\title{
Phase-field modeling of eutectic growth
}

\author{
François Drolet, ${ }^{1}$ K. R. Elder, ${ }^{2}$ Martin Grant, ${ }^{3}$ and J. M. Kosterlitz ${ }^{4}$ \\ ${ }^{1}$ Supercomputer Computations Research Institute, Florida State University, Tallahassee, Florida 32306-4052 \\ ${ }^{2}$ Department of Physics, Oakland University, Rochester, Michigan 48309-4401 \\ ${ }^{3}$ Physics Department, Rutherford Building, McGill University, 3600 rue University, Montréal, Québec, Canada H3A $2 T 8$ \\ ${ }^{4}$ Department of Physics, Brown University, Providence, Rhode Island 02912
}

(Received 9 February 1998; revised manuscript received 8 October 1999)

\begin{abstract}
A phase-field model of eutectic growth is proposed in terms of a free energy $\mathcal{F}$, which is a functional of a liquid-solid order parameter $\psi$, and a conserved concentration field $c$. The model is shown to recover the important features of a eutectic phase diagram and to reduce to the standard sharp-interface formulation of nonequilibrium growth. It is successfully applied to the study of directional solidification when the solid phase is a single or two phase state. The crystallization of a eutectic compound under isothermal conditions is also considered. For that process, the transformed volume fraction and $\psi$-field structure factor, both measured during numerical simulations, closely match theoretical predictions. Three possible growth mechanisms are also identified: diffusion-limited growth, lamellar growth, and spinodal decomposition.
\end{abstract}

PACS number(s): 64.70.Dv, 05.70.Fh, 82.20.Mj, 05.70.Ln

\section{INTRODUCTION}

The ability to generate forms or patterns is a remarkable property of macroscopic systems. Understanding the principles at the origin of these patterns represents a formidable task to which numerous efforts have been devoted. Over the last 30 years considerable progress has been made mainly through the study of simple problems involving fluid flow or solidification processes. Classic examples of pattern formation in those fields include the onset of convective rolls in Rayleigh-Bénard cells [1-4] and the dendritic instability of solidification fronts [5-9]. Various models have been proposed to describe these phenomena, while experiments have helped determine the precise conditions under which they take place.

For processes involving liquid-solid transitions the simplest theoretical description is provided by the so-called minimal model of solidification $[6,10]$. It consists of one or several diffusion equations governing the transport of latent heat and/or chemical species through the system. Boundary conditions at the sharp liquid-solid interface and at infinity complete the model. Hydrodynamic and elastic effects are not considered. The apparent simplicity of the approach is somewhat deceptive as exact solutions have been found in only a few special cases (e.g., when the solidification front is planar or spherical [6]). Furthermore, extensive numerical work has been hampered by the difficulties involved in tracking the position of the interface $[11,12]$.

These difficulties have lead to the formulation of the phase-field approach $[13,14]$, in which information about the position of the interface is contained in the spatial dependence of some order parameter $\psi$ which assumes different values in the liquid and solid phases. The interfacial region over which $\psi$ changes from one value to the other has a thickness of the order of an equilibrium correlation length. The equation obeyed by the order parameter is presented in terms of a mesoscopic free energy functional $\mathcal{F}$. This describes the basic thermodynamics involved in the process, such as the existence of both liquid and solid phases, as well as their relative thermal and mechanical stability. The free energy functional is coupled to one or more diffusionlike equations, which govern the release and transport of latent heat and/or chemical species. The model thus consists of a small number of differential equations that are easily solved numerically.

This paper contains a detailed analysis of a recently introduced [15-17] phase-field model describing eutectic behavior. Examples of eutectic compounds include simple binary alloys such as $\mathrm{Pb}-\mathrm{Sn}$ [18] and $\mathrm{Mg}-\mathrm{Al}$ [19] and complex organic materials such as carbon tetrabromidehexachloroethane [20]. All have similar phase diagrams, the complexity of which allows for a number of different solidification processes. Results from our study of some of these processes are presented in this paper, which is structured as follows. The free energy functional $\mathcal{F}$ central to the model is introduced and analyzed using mean-field theory in Sec. II. In Sec. III, the phase-field model is shown to reduce to the classical formulation of the problem in the sharp-interface limit $[15,21-26]$. The process of lamellar eutectic growth is analyzed in Sec. IV, with the emphasis on the wavelength selection problem and the various instabilities observed. The cellular instability characteristic of directional solidification is also recovered. Results from a numerical study of isothermal eutectic growth are presented in Sec. V. The process involves constant nucleation and growth rates, allowing theoretical predictions for the transformed volume fraction and $\psi$-field structure factor. The various growth mechanisms governing compositional segregation are also identified. Some technical details are given in Appendices A and B.

\section{MODEL}

The mesoscopic model is formulated in terms of a Ginzburg-Landau free energy $\mathcal{F}$ which is a functional of two coupled order parameters: a nonconserved liquid-solid order parameter $\psi$ and a conserved concentration field $c \propto C$ 


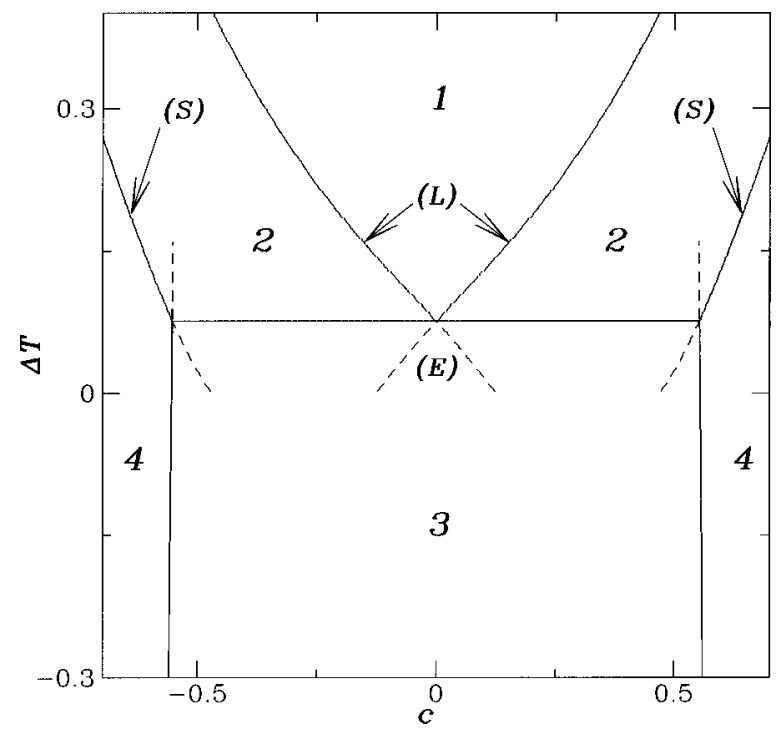

FIG. 1. Mean-field phase diagram in the $(c, \Delta T)$ plane corresponding to $f(\psi, c)$ with the parameter set $(r, a, b, w, \alpha, \beta)$ $=(1,1,1,0,0.15,0.15)$. Solid lines separate the various regions of the phase diagram [1 (liquid), 2 (solid-liquid coexistence), 3 (solidsolid coexistence), and 4 (single phase solid)]. The dashed lines represent metastable extensions of these boundaries. $(S)$ is the solidus and $(L)$ the liquidus. $(E)$ denotes the eutectic point.

$-C_{E}$, where $C_{E}$ is the eutectic composition. The thermodynamic properties to be accounted for are summarized in the eutectic phase diagram displayed in Fig. 1. At high temperatures, (region 1) the equilibrium state of the system consists of a liquid of uniform composition. Coexistence between a liquid and a solid is possible inside the two sidearms corresponding to region 2 . Each arm is delimited by liquidus $(L)$ and solidus $(S)$ curves. The two liquidus lines meet at the eutectic point $(E)$, where three-phase coexistence is possible: a liquid of eutectic composition coexists with two solids rich in either component (denoted $A$ and $B$, respectively). At that point the free energies associated with the three phases are all equal. As temperature is lowered the liquid becomes metastable and eventually solidifies. Depending on the average composition of the sample, the liquid either transforms into a mixture of the two solids (region 3) or becomes a solid of uniform composition (region 4). From these features we can anticipate the appropriate form for the free energy $\mathcal{F}$.

The free energy is the sum of local parts which interact via, for example, a square-gradient term. Since it is a mesoscopic model, there are no nonanalytic pieces; any such dependence can only arise in the thermodynamic free energy $-k_{B} T \ln \Sigma_{\psi, c} e^{-\mathcal{F} / k_{B} T}$, where $k_{B}$ is Boltzmann's constant, and $T$ is temperature. First $\mathcal{F}$ must allow two-phase coexistence. A convenient form is the double-well potential $f_{\psi}=-\psi^{2}$ $+\psi^{4}+\nu \psi$. This form reflects the existence of both liquid and solid phases, with the coefficient $\nu$ determining their relative stability. As a convention, a positive (negative) value of $\psi$ is identified with the solid (liquid) phase. Thus $\nu$ must be positive at high temperatures and negative otherwise. The free energy must also account for the fact that the concentration is homogeneous in the liquid phase and can separate in the solid phase. This is accomplished by adding the form $f_{c}=-\psi c^{2}+c^{4}$, in which the sign of $\psi$ determines whether the $c$ field phase separates $(\psi>0)$ or not $(\psi<0)$. With these considerations, the general form of $\mathcal{F}$ can be written

$$
\mathcal{F}\{c, \psi\}=\int d \vec{x}\left[f(c, \psi)+\frac{K_{\psi}}{2}|\vec{\nabla} \psi|^{2}+\frac{K_{c}}{2}|\vec{\nabla} c|^{2}\right],
$$

where

$$
f=-\frac{r}{2} \psi^{2}+\frac{a}{4} \psi^{4}+\left(\alpha \Delta T-\beta c^{2}\right) \psi+\frac{w}{2} c^{2}+\frac{b}{4} c^{4} .
$$

Note that $\mathcal{F}\{c, \psi\}$ is an effective free energy describing the system on a coarse-grained scale. Processes on smaller length scales have already been averaged over and incorporated into parameters such as $r$ and $a$. The local part of the free energy functional $f$ involves the positive parameters $r, a$, $\alpha, \beta, w$, and $b$, where $w<2 \beta$, and the excess temperature $\Delta T=T-T_{m}$, where $T_{m}$ is the melting temperature at $\beta=0$ (not the eutectic temperature $T_{E}$ ). Mean-field theory will be used below to relate these parameters to thermodynamic quantities. The terms $K_{\psi}|\vec{\nabla} \psi|^{2} / 2$ and $K_{c}|\vec{\nabla} c|^{2} / 2$ are included in order to account for the energetic cost associated with the presence of interfaces in the system. Note also that for the mesoscopic model to be well defined it is implicit that there be an ultraviolet cutoff in integrals over space. That is, integrals over $\vec{x}$ are restricted to $|\vec{x}|>l_{0}$, where $l_{0}$ is a small length scale [27], which may be thought of as the lattice constant.

The dynamics used in this paper are the simplest possible dissipative dynamics which drives the system toward thermodynamic equilibrium, subject to external constraints, such as some specified temperature distribution, and which respects the conservation of $c$. The effects of flows in the high temperature liquid phase are not considered. Inclusion of these requires an additional field describing the momentum density of the liquid in the free energy $\mathcal{F}$, a dynamical Navier Stokes equation for its dynamics, and various mode coupling terms in the dynamics for $\psi$ and $c$. The dynamics of $\psi$ and $c$ are given by the Langevin equations

$$
\frac{\partial \psi}{\partial t}=-\Gamma_{\psi} \frac{\delta \mathcal{F}}{\delta \psi}+\eta_{\psi}
$$

and

$$
\frac{\partial c}{\partial t}=\Gamma_{c} \nabla^{2} \frac{\delta \mathcal{F}}{\delta c}+\eta_{c}
$$

The mobilities $\Gamma_{c, \psi}$ are constant [28], while thermal fluctuations obey the fluctuation-dissipation relations $\left\langle\eta_{\psi}(\vec{x}, t) \eta_{\psi}(0,0)\right\rangle=2 \Gamma_{\psi} k_{b} T \delta(\vec{x}) \delta(t)$ and $\left\langle\eta_{c}(\vec{x}, t) \eta_{c}(0,0)\right\rangle$ $=2 \Gamma_{c} k_{b} T \nabla^{2} \delta(\vec{x}) \delta(t)$. In this paper, analysis is limited to cases in which the temperature $\Delta T$ is fixed externally. This is an excellent approximation for two-dimensional films, metals, and metalloids where concentration diffusion is orders of magnitude slower than heat diffusion. Results obtained using a modified eutectic model which includes latent heat generation at the interface and subsequent diffusion through the system can be found in Ref. [17]. 


\section{Phase diagram}

The phase diagram shown in Fig. 1 was derived from a mean-field analysis of the model

$$
-k_{B} T \ln \sum_{\psi, c} e^{-\mathcal{F} / k_{B} T} \approx \mathcal{F}_{\text {mean field }}
$$

where $\mathcal{F}_{\text {mean field }}$ is the free energy functional evaluated at the extrema of $c$ and $\psi$. It was obtained by first minimizing the bulk free energy $f(c, \psi)$ with respect to $\psi$. Provided $\mid \alpha \Delta T$ $-\beta c^{2} \mid / 2 a \ll(r / 3 a)^{3 / 2}$, the solutions to $\partial f(\psi, c) / \partial \psi=0$ are

$$
\psi_{L} \approx-\left(\frac{r}{a}\right)^{1 / 2}-\frac{1}{2 r}\left(\alpha \Delta T-\beta c^{2}\right)
$$

for the liquid phase, and

$$
\psi_{S} \approx+\left(\frac{r}{a}\right)^{1 / 2}-\frac{1}{2 r}\left(\alpha \Delta T-\beta c^{2}\right),
$$

for the solid phase.

Substituting $\psi_{L}$ and $\psi_{S}$ into $f(\psi, c)$ defines two functions of $c, f_{L}(c)$ and $f_{S}(c)$ [see Fig. 2(a)]. The equilibrium state of the system is then specified by imposing a uniform chemical potential $\left(\mu \equiv \delta \mathcal{F} / \delta c=\mu_{0}\right)$. The various lines on the phase diagram are recovered by assuming this equilibrium state consists of two phases of respective composition $c_{1}$ and $c_{2}$, separated by a planar interface at $z=0$. Letting $c \equiv c(z)$, the condition $\mu_{0}=\delta \mathcal{F} / \delta c$ becomes $K_{c} d^{2} c / d z^{2}=\partial f(\psi, c) / \partial c$ $-\mu_{0}$. This simplifies by integrating both sides over $c$ and noting that the left-hand side identically vanishes. Hence

$$
\int_{c_{1}}^{c_{2}} d c\left(\frac{\partial f}{\partial c}-\mu_{0}\right)=f\left(c_{2}\right)-f\left(c_{1}\right)-\left(c_{2}-c_{1}\right) \mu_{0}=0
$$

with $\partial f /\left.\partial c\right|_{c_{1}, c_{2}}=\mu_{0}$. Equation (8) is Maxwell's equal-areaconstruction rule, which determines $c_{1}, c_{2}$, and $\mu_{0}$ [see Fig. 2(b)]. The entire phase diagram is recovered by probing a wide range of temperatures. Metastable extensions of the liquidus and solidus lines (included in Fig. 1) are associated with the existence of a second set of solutions to Eq. (8). Near the eutectic point it is possible to obtain analytic expressions for many important quantities such as the concentration on the coexistence lines, the eutectic temperature, the chemical potential, and the slopes of the liquidus and solidus lines. These are given below.

Note that the symmetry of the phase diagram reflects the invariance of the particular form of $f$ under $c \rightarrow-c$. Other forms of $f$ can be chosen which are not symmetric. The present convenient choice highlights generic features of eutectic growth. It should also be noted that the liquidus and solidus lines can be made to converge at $c= \pm 1$, corresponding to a pure $A$ or pure $B$ sample, through the use of an alternate form of $f$, such as $f(\psi, c)=-r \psi^{2} / 2$ $+a \psi^{4} / 4+\left(\alpha \Delta T-\beta c^{2}\right) \psi+\omega c^{2} / 2+(3 b / 2)[(1-c) \ln (1-c)$ $\left.+(1+c) \ln (1+c)-c^{2}\right]$. Finally, as shown by Kobayashi [29], for the study of dendritic instabilities it can be of use to break the $\pm \psi$ symmetry with the alternate term $(\alpha \Delta T$ $\left.-\beta c^{2}\right) \Psi(\psi)$, where $\Psi( \pm \psi)= \pm \Psi$ is chosen such that $d \Psi /\left.d \psi\right|_{e q}=0$.
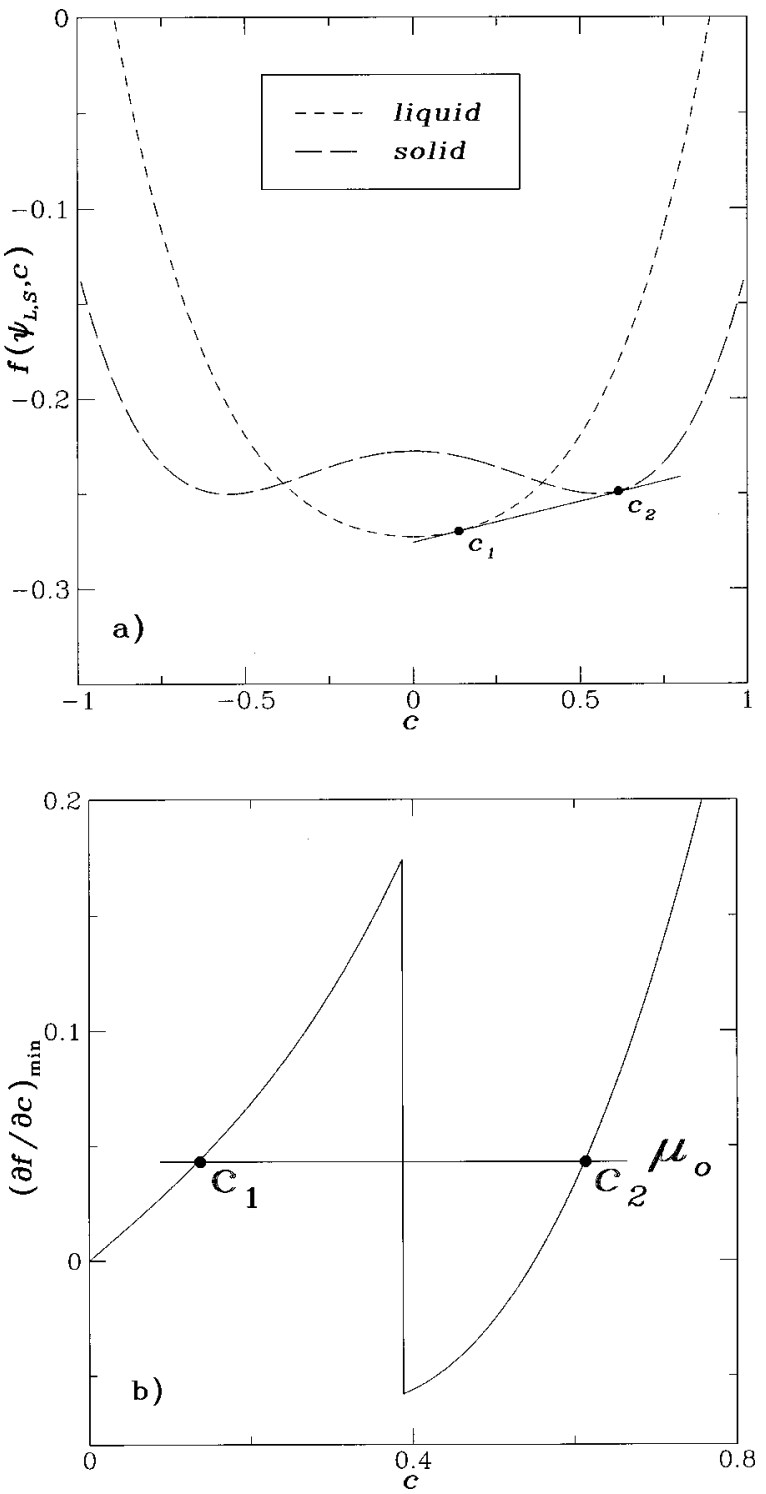

FIG. 2. (a) The compositional dependence of $f$ for either the liquid or solid phase. The equilibrium compositions can be determined using the common tangent construction. (b) One can also find the value $\mu_{0}$ such that the integral of $(\partial f / \partial c)_{\text {min }}$ between $c_{1}$ and $c_{2}$ vanishes. The example shown has $\Delta T=0.15$, with the other parameters assuming the values given at the bottom of Fig. 1 .

\section{SHARP-INTERFACE LIMIT}

The preceding mean-field treatment indicates that the free energy accounts for the various regions characteristic of a eutectic phase diagram. This is one of the useful features of this approach involving the Ginzburg-Landau free energy: Mean-field theory is simple and transparent. The validity of the phase-field model can be further established by showing that it reduces to the classical sharp-interface formulation of eutectic growth. This will again be done by mean-field theory.

However, some caution should be used to avoid overinterpreting this mean-field limit. It is well known that the actual values of the parameters in the Ginzburg-Landau free energy functional can have little direct physical meaning. Formally, observables such as the correlation length, specific heat, diffusion coefficients, and so on are particular limits of 
space and time dependent response functions. In particular, the interface between two phases (liquid-solid or solid-solid) involves a thickness $\xi$ which can be a complicated function of all the parameters of the underlying microscopic theory. Roughening of an interface and critical phenomena are two examples of this behavior [27]. As mentioned above, for the Ginzburg-Landau description to be valid on all length scales, one must incorporate an ultraviolet cutoff $l_{0}$, which is a microscopic length scale of order of the interparticle distance. To give one example, above the roughening transition, a three-dimensional surface has a contribution to its width, in addition to intrinsic width, due to roughening, which is proportional to $\sqrt{\ln \left(L / l_{0}\right)}$, where $L$ is the edge length of the system. The "sharp-interface" limit corresponds to the wellordered set of limits: $l_{0} \rightarrow 0^{+}, \quad \xi / l_{0} \rightarrow \infty$, and $\xi / R \ll 1$, where $R$ is a macroscopic length scale. An advantage of the present method is that $\xi$ can be appreciable so long as $R$ is kept significantly larger, with $\xi / R \ll 1$ satisfied. This is numerically convenient, since this can be satisfied to a less stringent tolerance than experimental systems, where one can have $\xi / R \sim 10^{-8}$. In addition, note that the naive limits of $l_{0}=0$, with $\xi / R \rightarrow 0^{+}$accomplished by $\xi \rightarrow 0$ are well known to be potentially inconsistent and unphysical.

A consistent method to obtain the macroscopic sharpinterface limit is given in Appendix B below, where an expansion around a stationary planar interface [30] is given. The equations recovered are (i) the diffusion equation governing changes in composition in the bulk,

$$
\frac{\partial c}{\partial t}=D_{L, S} \nabla^{2} c
$$

where $D_{L, S}$ is the diffusion constant in the liquid $(L)$ or solid $(S)$ phase; (ii) the Gibbs-Thomson condition describing how local curvature affects the composition on both sides of an interface,

$$
\frac{\delta c}{\Delta c_{m i s}}=d_{0} \kappa+\frac{\zeta}{l_{T}}+\beta_{v} v_{n},
$$

where $\Delta c_{m i s}$ is the miscibility gap, $d_{0}$ is the capillary length, $\kappa$ is the curvature, $l_{T}$ is the thermal length, $\zeta$ is the shortest distance between the liquid-solid interface and the eutectic temperature, and the last term on the right-hand side, involving the kinetic coefficient $\beta_{v}$ and the normal velocity $v_{n}$, gives the kinetic undercooling, (iii) the conservation law relating the flow of $c$ across an interface to the local velocity of that interface;

$$
v_{n} \Delta c=\left.D_{L} \frac{\partial c}{\partial u}\right|_{L}-\left.D_{S} \frac{\partial c}{\partial u}\right|_{S}
$$

and (iv) the condition of mechanical equilibrium which determines the various angles between interfaces when the three phases meet

$$
\theta=\sin ^{-1}\left(\sigma_{A B} / 2 \sigma_{L S}\right),
$$

where $\sigma_{A B}$ and $\sigma_{L S}$ are the surface tension at a solid-solid and liquid-solid interface, respectively.

Of course, the analysis does more than permit one to recover the macroscopic sharp-interface description. In addi-

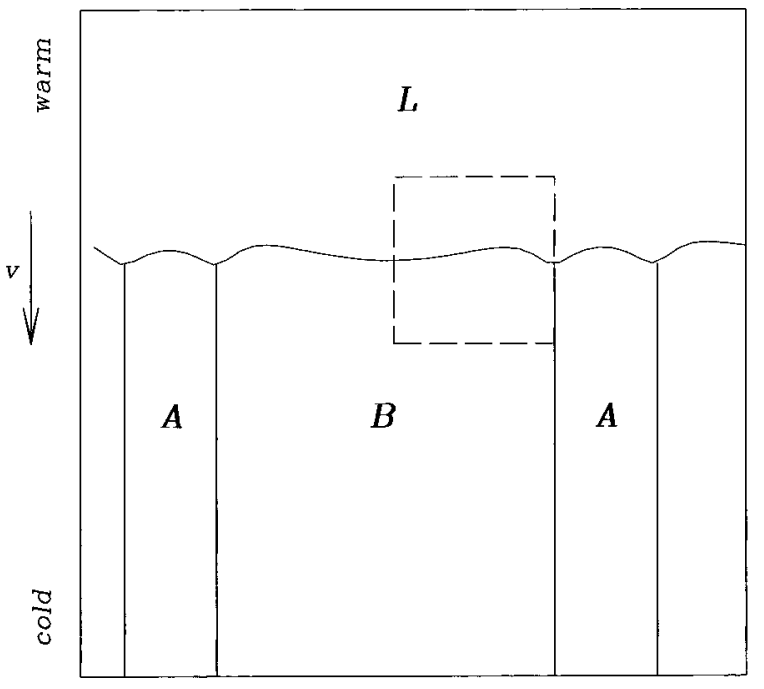

FIG. 3. Steady-state lamellar eutectic growth. The lamellae grow upwards with a velocity equal in magnitude to the pulling velocity $v$. The configuration shown was obtained from the phasefield model at an average composition $c_{0}=0.4$. Vertical lines correspond to locations where $c=0$, while the liquid-solid interface consists of points where $\psi=0$. Dashed lines show the portion of liquid-solid interface with small local curvature.

tion, one can recover explicit forms for the various parameters entering into that description, in terms of the parameters of the underlying theory, such as the surface tension.

\section{DIRECTIONAL GROWTH}

In this section the eutectic phase-field model is used to study directional solidification when the emerging solid contains two phases of different concentration (Sec. IV A) or one phase (Sec. IV A). Both processes involve pulling a liquid at some constant velocity $v$ through a temperature gradient, leading to the formation of a solidification front perpendicular to the pulling direction.

\section{A. Two phase directional solidification}

For two solid phases to emerge the system is pulled through a temperature gradient $\Delta T=G z$ at a constant velocity, such that the average composition of the system lies inside the solid-solid coexistence region of the phase diagram at low temperatures. The system reacts to the temperature gradient by forming lamellar or rodlike structures of the $A$-rich and $B$-rich phases [31] in the solid region. The steadystate configuration shown in Fig. (3) consisting of alternating lamellae of $A$ - and $B$-rich phases is typical of lamellar growth. These lamellae grow at a constant speed $v$ equal in magnitude but opposite in direction to the external pulling velocity. The average composition of the liquid determines their relative width.

Configurations such as this one were obtained using a discrete map of Eqs. (3). Explicitly, in a frame of reference moving at speed $v$ in the $z$ direction (along index $j$ in the discretized equation), 


$$
\begin{aligned}
\psi_{i, j}(n+1)= & \psi_{i, j}(n)+\Gamma_{\psi} \Delta t\left[r \psi_{i, j}(n)-a \psi_{i, j}^{3}(n)\right. \\
& +\left(\beta c_{i, j}^{2}(n)-\alpha \Delta T_{j}\right)+K_{\psi} \mathcal{L} \psi_{i, j}(n) \\
& \left.+\frac{v\left(\psi_{i, j+1}-\psi_{i, j}\right)}{\Delta x}\right]
\end{aligned}
$$

and

$$
\begin{aligned}
c_{i, j}(n+1)= & c_{i, j}(n)+\Gamma_{c} \Delta t\left\{\mathcal { L } \left[w c_{i, j}(n)-2 \beta c_{i, j}(n) \psi_{i, j}(n)\right.\right. \\
& \left.\left.+b c_{i, j}^{3}(n)-K_{c} \mathcal{L} c_{i, j}(n)\right]+\frac{v\left(c_{i, j+1}-c_{i, j}\right)}{\Delta x}\right\},
\end{aligned}
$$

where the spatial operator $\mathcal{L}$ is the discrete equivalent of a Laplacian. In all simulations this operator was determined by consideration of nearest neighbors only, for example on a square lattice $\quad \mathcal{L} \psi_{i, j} \equiv\left(\psi_{i+1, j}+\psi_{i, j+1}+\psi_{i-1, j}+\psi_{i, j-1}\right.$ $\left.-4 \psi_{i, j}\right) / \Delta x^{2}$. The indices $n$ and $(i, j)$ can be used to recover space and time units, through the relationships $t=n \Delta t$ and $\vec{x}=(i \hat{x}+j \hat{z}) \Delta x$.

Steady-state configurations were obtained by iterating Eqs. (13) and (14) starting from initial conditions of the form $\psi_{i, j}=\epsilon_{i, j}$ and $c_{i, j}=c_{0}+\epsilon_{i, j}$, where $\epsilon_{i, j}$ are independent random variables uniformly distributed between $-1 / 2$ and $+1 / 2$. Various system sizes were used, with the length $L_{z}$ of the simulation box in the $\hat{z}$ direction chosen according to the average composition and pulling velocity. Liquids of offeutectic composition require larger values of $L_{z}$, as a boundary layer then forms in front of the interface. Periodic boundary conditions were used in $x$, while the derivatives of both fields were set to zero at the top and bottom of the simulation box. At a given average composition the selected wavelength $\lambda$ (corresponding to the total width of two neighboring lamellae) was found to be a decreasing function of the pulling velocity $v$. This is a well-known result that reflects the fact that the faster the growth velocity the less time atoms have to diffuse and thus, the closer neighboring lamellae must be. A quantitative understanding of the relationship between $v$ and $\lambda$ is due to Jackson and Hunt [21], who solved the steady-state diffusion equation and boundary conditions (see Sec. III), by perturbing around a planar interface. For any given pulling velocity they found an infinite number of solutions, each one corresponding to a different lamellar spacing. To remove this ambiguity, which is not present experimentally, Jackson and Hunt formulated the so-called minimum undercooling hypothesis in which the selected wavelength at a given velocity $v$ is assumed to minimize the undercooling so that growth takes place at an extremum. They obtained the well-established $[18,19]$ results $\lambda^{2} v$ $=$ const and $\left(\overline{\Delta T}-\Delta T_{E}\right)^{2} / v=$ const, where $\overline{\Delta T}$ is the average undercooling of the interface. A rigorous derivation or fundamental understanding of these two results is still lacking.

It is interesting to note that the phase-field approach illustrates transparently a relationship between $\lambda^{2} v=$ const and spinodal decomposition, as described by the Cahn-Hilliard
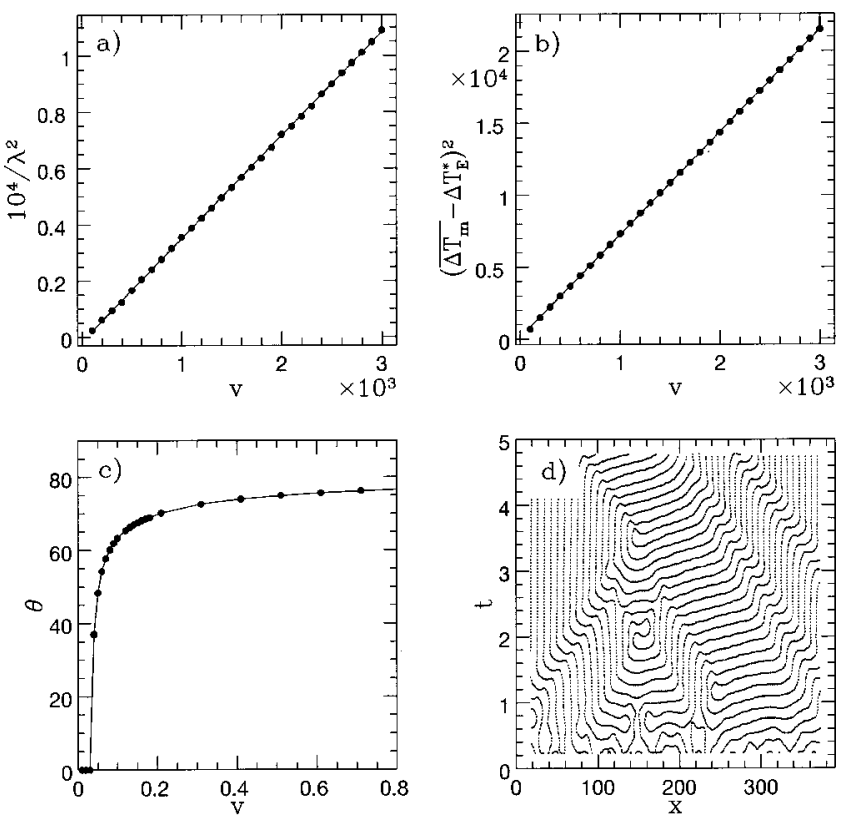

FIG. 4. (a) and (b) verify the Jackson and Hunt relationships for the dependence of lamellar spacing and average interfacial undercooling on pulling velocity in steady-state directional eutectic growth. In (c), the tilt angle $\theta$ is shown as a function of pulling velocity $v$. In (d), the solid-solid interface positions just behind the liquid-solid front are shown as a function of time. Several "tilt waves" are apparent. $c_{0}=0$ for all simulations shown here.

equation $[32,33]$. During spinodal decomposition, there is a dominant time-dependent length, to which other lengths scale, which obeys $L \sim t^{1 / 3}$ [34-37]. If one imposes a weak temperature gradient on the Cahn-Hilliard equation, and pulls with velocity $v$, a lamellar structure of wavelength $\lambda$ is eventually formed. Since the dominant length during the formation of the lamellae follows the scaling of spinodal decomposition, one obtains $\lambda^{2} v=$ const, from dimensional analysis, which is the same criterion as the Jackson-Hunt result. The connection of spinodal decomposition to eutectics, in an imposed velocity-dependent temperature gradient, is that the present phase-field model of eutectics reduces formally to the Cahn-Hilliard model of spinodal decomposition in that particular case. In addition, this suggests a simple crossover from an early time behavior dominated by domain growth to a regime of steady-state lamellar growth. Dimensional analysis implies the equivalent scaling forms $v$ $\sim\left(t^{1 / 3}\right)^{-2} f(l)$ or $v \sim \lambda^{-2} g(l)$, with $l \equiv \lambda / t^{1 / 3}$ and $g(l)$ $=l^{2} f(l)$. Provided $f(l \rightarrow \infty)=1$ and $g(l \rightarrow 0)=1$, these two forms describe a crossover from Ostwald ripening for early times or large $\lambda$ to a regime in which $\lambda^{2} v=$ const for late times or small $\lambda$.

To determine quantitatively the relationship between $\lambda$ and $v$ using the phase-field approach, a second set of simulations were performed in which the minimum undercooling hypothesis was used as a selection mechanism. In these simulations, done at eutectic composition $c_{0}=0$, the system was prepared in a lamellar structure of varying wavelength. Equations (13) and (14) were iterated until a steady state was reached. The shape of the interface separating the lamellae from the liquid was then found and the average undercooling calculated. The lamellar spacing with the lowest average un- 

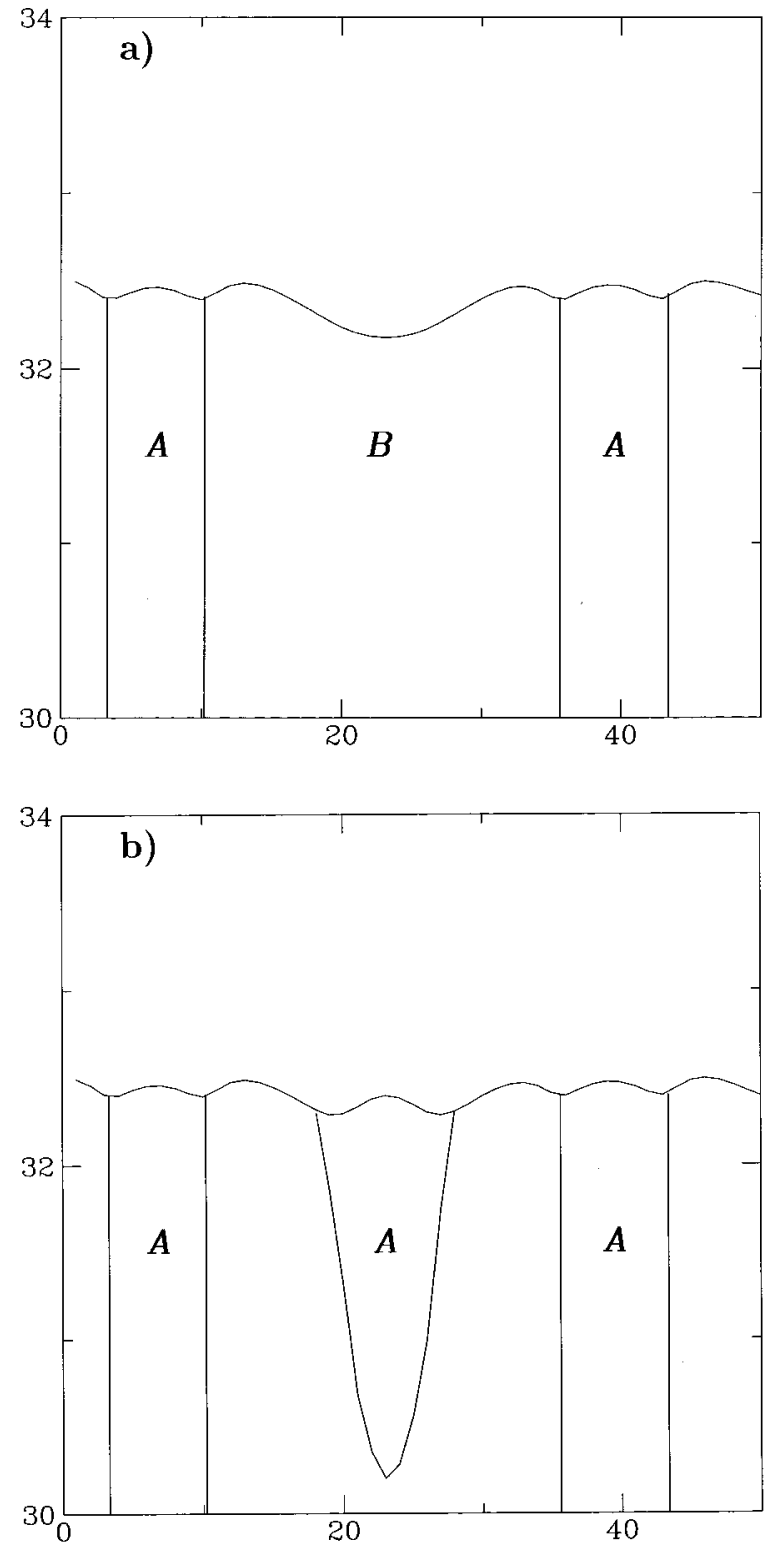

FIG. 5. Shape instability in lamellar growth. The configuration displayed in Fig. 3 was used as an initial condition. The velocity was increased from $v=0.05$ to $v=0.08$ over a period $\tau=5$. The two configurations shown correspond to $t=87.5$ (a) and $t=122.5$ (b). Again, the liquid-solid profile consists of points where $\psi=0$, while the other lines correspond to zeros of the $c$-field.

dercooling was identified with the selected wavelength. Figure 4(a) shows the dependence of the selected lamellar spacing on the pulling velocity $v$. As expected, the $\lambda^{-2} \propto v$ relationship was found to hold for a wide range of velocities confirming the predictions of the minimum undercooling hypothesis. The equivalent form $\left(\overline{\Delta T}-\Delta T_{E}\right)^{2} \propto v$ was also verified [Fig. 4(b)]. Interestingly, different branches of solutions were obtained using different initial conditions such as different positions of the interface at $t=0$. Kassner and Misbah [25] found similar results using a boundary integral formulation of the process.

Two different instabilities characteristic of lamellar eutectic growth were also observed in numerical simulations of our model. The first instability, present mainly at off-eutectic compositions $c_{0} \neq 0$, is illustrated in Fig. 5. The configura-

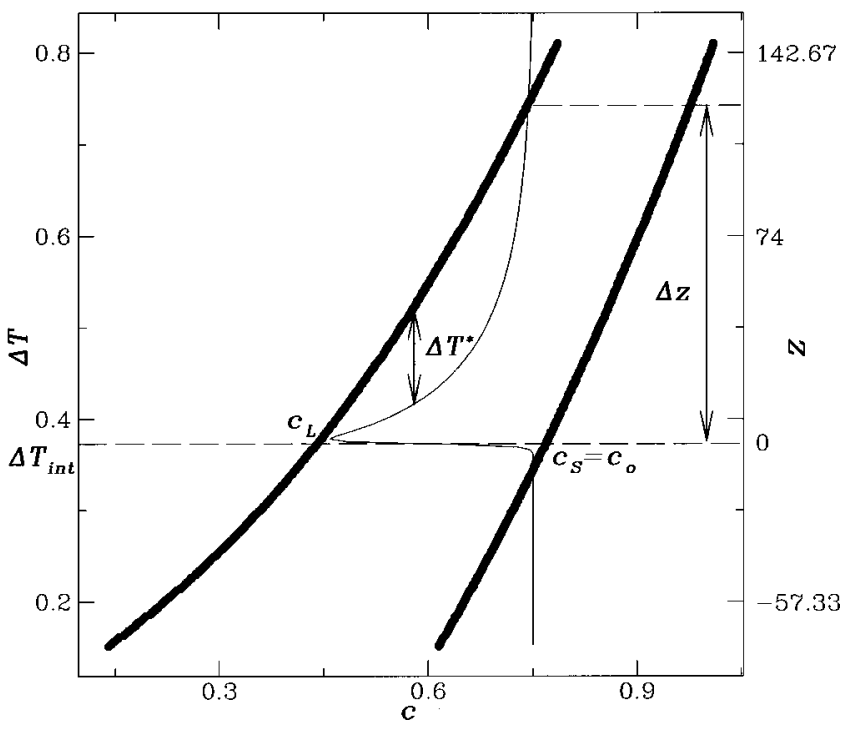

FIG. 6. Schematic representation of directional solidification. The profile in composition $c(z)$ (thin solid line) was obtained from a one-dimensional simulation of the process (with $v=0.05$ and $c_{0}$ $=0.75$ ). The liquidus and solidus lines (thick solid lines) are those of the phase diagram displayed in Fig. 1. Constitutional supercooling is present when the profile in composition lies inside the liquidsolid coexistence region (e.g., between $z=0$ and $z=\Delta z$ in the figure). At a given composition, the distance between the actual temperature and that on the liquidus gives the size of the supercooling (e.g., $\Delta T^{*}$ at $c=0.58$ ).

tions displayed were obtained from that of Fig. 3 by progressively increasing the velocity from $v=0.05$ to 0.08 , where $c_{0}=0.4$. The wider phase reacts to the increase by forming a pocket that progressively drops back as the growth proceeds. The interface eventually becomes unstable, leading to the appearance of a new lamella of the opposite phase. The net result of the event is a large local reduction of the lamellar spacing. This mechanism is expected to play an important role when both the jump in velocity, and the difference in width between the two phases are sufficiently large. The simulations also show the occasional occurrence of tilt domains in which lamellae grow at an angle $\theta$ with the vertical. This parity-breaking instability, predicted by Karma [24] and Kassner and Misbah [26] was observed in the lamellar growth of the transparent alloy $\mathrm{CBr}_{4}-\mathrm{C}_{2} \mathrm{Cl}_{6}$ by Faivre and co-workers $[38,39]$.

In the simulations of the phase-field model, tilted lamellae could be stimulated by a rapid increase in the velocity or by including thermal fluctuations in the dynamics. As in experiments, the instability either gives rise to solitary tilt waves $[38,39]$ which drift sideways through the system [Fig. 4(d)], or results in a homogeneous tilt of the entire lamellar structure. This transition from an untilted to a tilted state has been described as a tilt bifurcation [25,26], in which the tilt angle rises sharply from zero at some finite threshold velocity $v_{t}$. Results from simulations, performed without thermal fluctuations, are consistent with this interpretation [Fig. 4(c)].

\section{B. One phase directional solidification}

In this case, the average composition $c_{0}$ is such that, at low temperature, the equilibrium phase is a homogeneous 


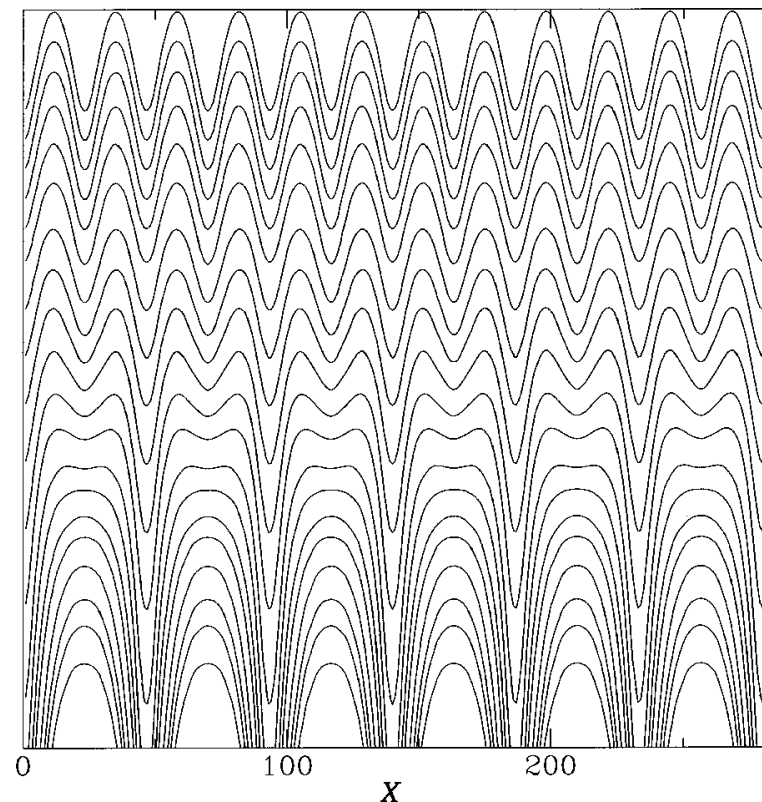

FIG. 7. Tip-splitting instability in directional solidification. The solidification front at the bottom of the figure corresponds to a steady-state profile with $c_{0}=1.08, \quad v=0.15, \quad$ and $\left(\Gamma_{c}, \Gamma_{\psi}, K_{\psi}, K_{c}, r, a, b, w, \alpha, \beta\right)=(0.5,1,1,1,1,1,1,0,1,0.5)$. Following a gradual increase in velocity from $v=0.15$ to 0.55 , each cell changes shape and eventually divides.

solid outside the two-phase coexistence region. Figure 6 shows the resulting composition profile perpendicular to the solidification front. The profile, obtained from a onedimensional simulation, corresponds to a steady state with liquid and solid phases fixed at each end of the sample, both of identical composition $c_{0}=0.75$. The interface between the two is in thermodynamic equilibrium with the points $\left(c_{L}, \Delta T_{i n t}\right)$ and $\left(c_{S}, \Delta T_{i n t}\right)$ lying, respectively, on the liquidus and on the solidus lines which are also included in the figure. The solute-rich boundary layer in the liquid forms during the initial transient and is at the origin of the cellular instability observed at large pulling velocities or small temperature gradient $G$. The driving force behind this instability is the so-called constitutional supercooling $[40,41]$ present if the actual temperature of the liquid is below the equilibrium liquidus temperature $\Delta T_{L}$. In the example displayed in Fig. 6 , the liquid is supercooled over a range $\Delta z$, with the supercooling equal to $\Delta T^{*}$ at $c=0.58$.

A planar interface becomes unstable when the front is constitutionally supercooled which occurs when $G / v$ $<m_{L}\left(c_{0}-c_{L}\right) / D_{L}$. This instability is observed in both transparent organic materials [42] and metallic systems [43]. In either case the initially flat interface reacts to sudden changes in temperature gradient or pulling velocity by transforming into a cellular structure. The characteristic size of this structure can be predicted by a stability analysis of the unperturbed planar front. A linear stability analysis of the sharp interface equations of Sec. III was performed by Mullins and Sekerka [44].

Note that a phase-field model specifically designed to describe directional solidification has been proposed [45]. That model can be obtained by expanding Eqs. (1)-(4) to the
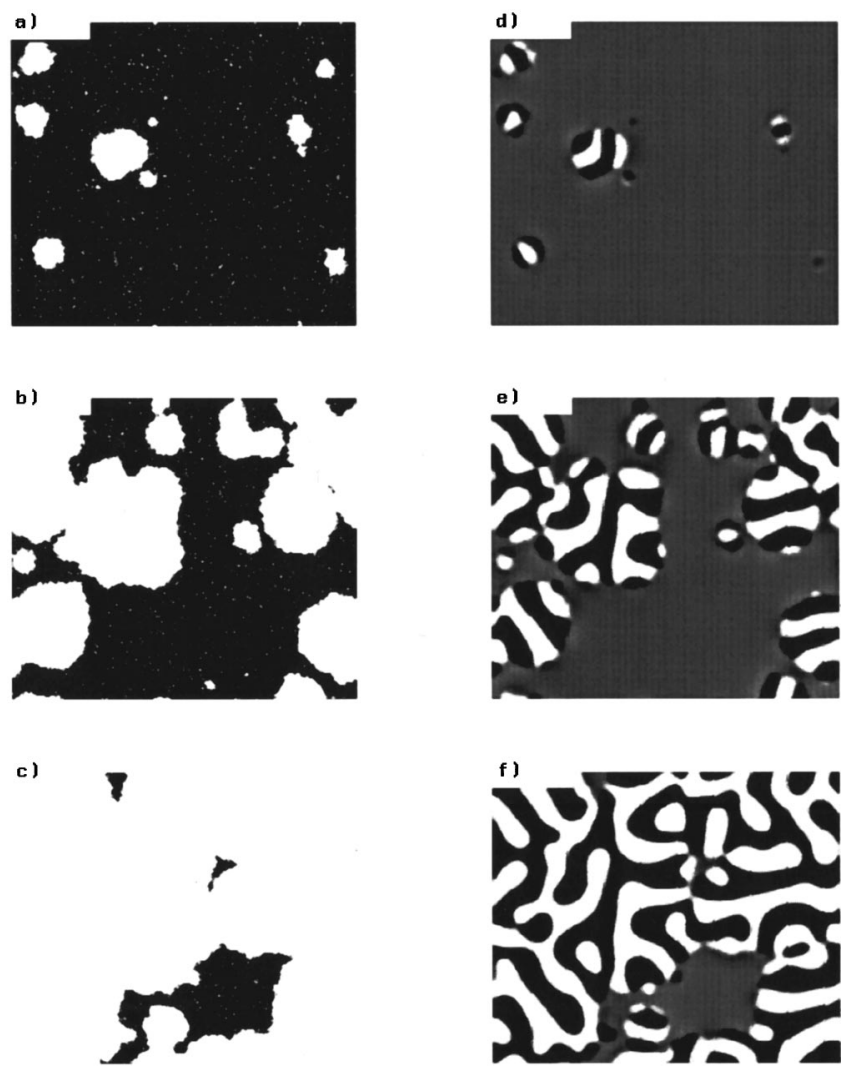

FIG. 8. Gray-scale representations of the liquid-solid order parameter $\psi$ (left column) and the concentration field $c$ (right column) with $c_{0}=0.0$. The configurations shown are at times $t=1350,2700$, and 4050 . In (a)-(c), regions where $\psi>0$ are in white and correspond to the solid phase. In (d)-(f), black and white regions correspond respectively to a solid rich in $A(c \approx-0.55)$ or in $B(c \approx$ $+0.55)$.

lowest nontrivial order in $\tilde{c}=c-c_{0}$. In Fig. 7 , we show a tip-splitting instability caused by an increase in velocity.

\section{ISOTHERMAL EUTECTIC GROWTH}

This section deals with the isothermal solidification of eutectic compounds. The study presented focuses on cases in which a liquid of composition $c_{0}$ and temperature $\Delta T$ is suddenly quenched to a lower temperature $\Delta T^{\prime}$ such that the point $\left(c_{0}, \Delta T^{\prime}\right)$ lies inside the solid-solid coexistence region of the phase diagram. The phase-field approach leads to an analysis of this problem in terms of two concurrent processes: (i) the nucleation and subsequent growth of solid droplets inside the metastable liquid; and (ii) the segregation of the two components inside the solid, leading to the two equilibrium concentrations (the $A$ - and $B$-rich solids) expected from the phase diagram. These processes are described by the Langevin equations presented earlier. The main results presented here are from extensive numerical simulations performed at a variety of average compositions $c_{0}=0.0,0.08,0.12$, and 0.16 . All simulations were done on a two-dimensional $256 \times 256$ lattice using periodic boundary conditions. A hexagonal lattice was chosen to minimize anisotropy effects. Equations (3) were integrated in the nearestneighbor approximation using a mesh size $\Delta x=1.3$ and a time step $\Delta t=0.05$. The temperature was set to $\Delta T=-0.4$ 

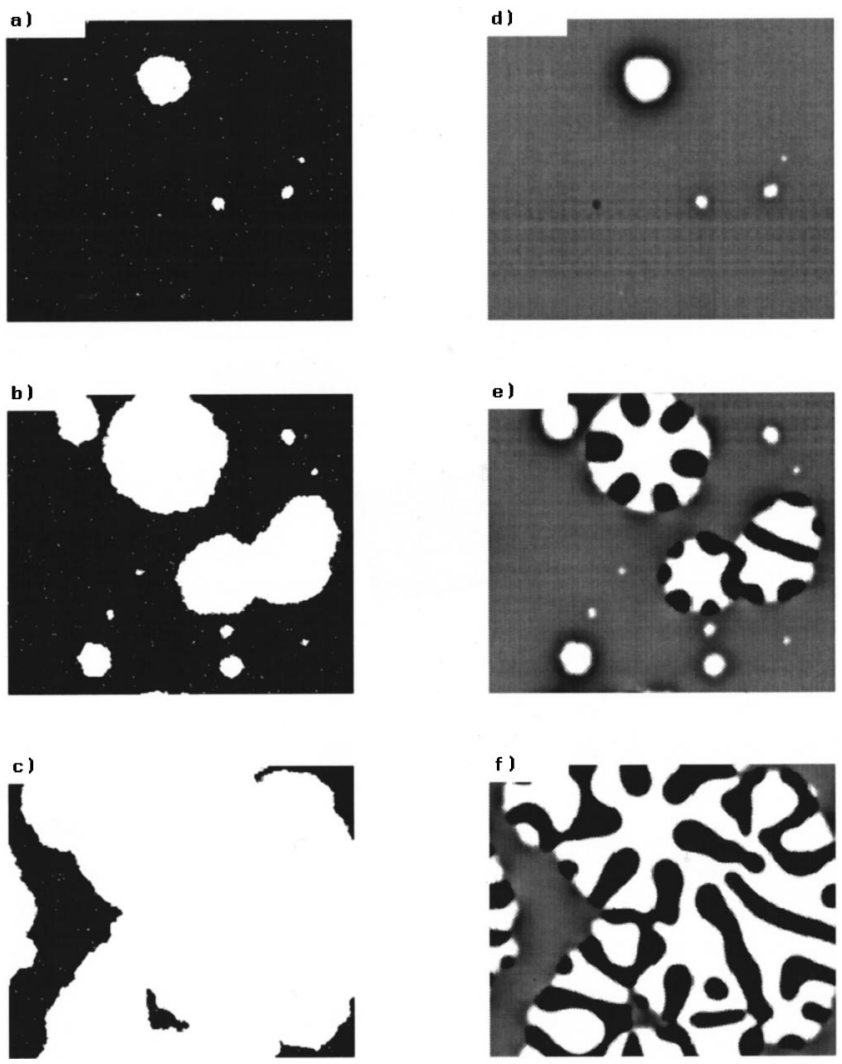

FIG. 9. Gray-scale representations of the liquid-solid order parameter $\psi$ (left column) and the concentration field $c$ (right column) with $c_{0}=0.08$. The configurations shown are at times $t=2250$, 4500, and 6750. In (a)-(c), regions where $\psi>0$ are in white and correspond to the solid phase. In (d)-(f), black and white regions correspond respectively to a solid rich in $A(c \approx-0.55)$ or in $B$ $(c \approx+0.55)$.

while the various parameters entering the model assumed the values $\quad\left(K_{\psi}, K_{c}, r, a, b, w, \alpha, \beta\right)=(1 / 8,1,1,1,1,0,0.15,0.15)$. The stochastic nature of the nucleation process was accounted for by including thermal fluctuations of magnitude $0.26\left(0.29\right.$ for the $c_{0}=0.0$ case $)$ in the equation for $\psi$. The simulations started from an undercooled liquid state and were terminated when the system was approximately $95 \%$ crystallized. The initial state was characterized by the field values $\psi_{i, j}=-1+\epsilon_{i, j}$ and $c_{i, j}=\epsilon_{i, j}$, where $\epsilon_{i, j}$ is an uncorrelated random number with $\left|\epsilon_{i, j}\right| \leqslant 0.1$.

Figures $8\left(c_{0}=0.0\right)$ and $9\left(c_{0}=0.08\right)$ show configurations of both the $\psi$ field (left column), showing the nucleation and subsequent growth of solid droplets in the liquid, and the $c$ field (right column), showing the existence of both $A$ - and $B$-rich solids inside the droplets at various instants during a typical run. It is clear from the right column of Fig. 9 that a liquid rich in $B$ always nucleates the $B$-rich solid first [46]. A quantitative analysis was made possible by monitoring the solid volume fraction $X(t)$ and the spherically averaged structure factors of both fields: $S_{\psi}(q, t) \equiv\left\langle|\psi(\vec{q}, t)|^{2}\right\rangle$ and $S_{c}(q, t) \equiv\left\langle|c(\vec{q}, t)|^{2}\right\rangle$. These quantities were averaged over 25 runs (33 in the $c_{0}=0.0$ case). Results pertaining to the $\psi$ field will be presented in Secs. V A and VB. The various growth mechanisms controlling the formation of $A$ - and $B$-rich phases are discussed in Sec. V C, and correlated with changes in the $c$-field structure factor.

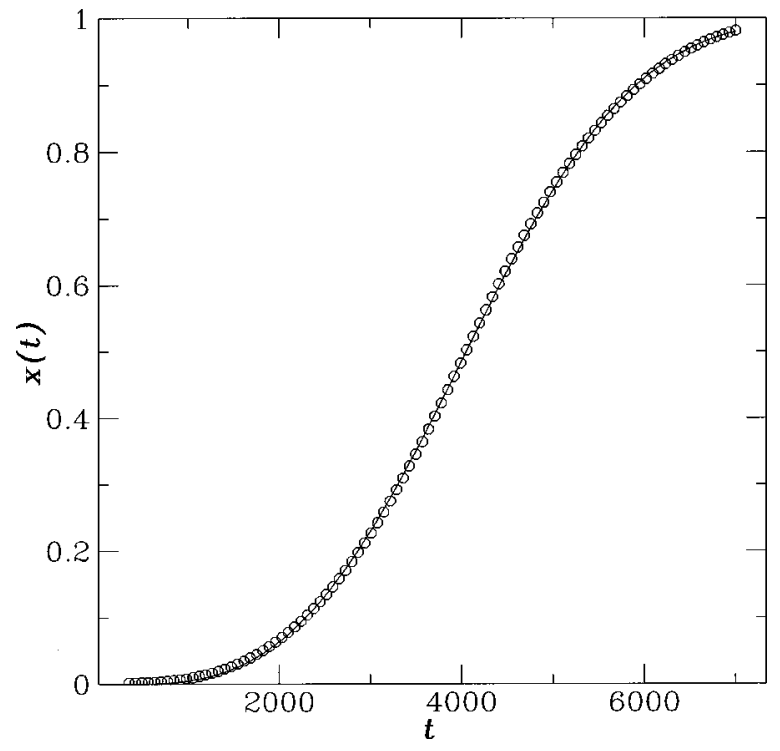

FIG. 10. Time dependence of the solid volume fraction $X(t)$ with $c_{0}=0.12$. The solid line passing through the data points is a fit to the Kolmogorov form [Eq. (15)]. The fit yielded the values $t_{0}$ $=276.9$ and $(\pi / 3) I v^{2}=1.29 \times 10^{-11}$.

\section{A. Transformed volume fraction}

The time dependence of the fraction of the liquid that has solidified can be obtained from purely geometric considerations if several simple assumptions are made. For homogeneous nucleation, with constant nucleation rate $I$ and droplet velocity $v$, the solid volume fraction $X(t)$ can be written

$$
X(t)=1-\exp \left\{-\frac{a_{d}}{d+1} I v^{d}\left(t-t_{0}\right)^{d+1}\right\}
$$

in $d$ dimensions, where $a_{2}=\pi, a_{3}=4 \pi / 3$, and $t_{0}$ is the waiting time [47-49]. It should be noted that this neglects the small dependence of the the droplet's growth velocity on curvature, and any initial time dependence of the nucleation rate. Numerically, the solid volume fraction was measured by periodically evaluating the fraction of lattice sites with $\psi>0$. The process was repeated during each run and the averaged transformed volume fraction $X(t)$ was fit to Eq. (15). Figure 10 shows both the data points and the fitted curve in the case $c_{0}=0.12$. Similar agreement was observed at all four average compositions. The agreement between simulation results and predictions from Eq. (15) is quite good considering the above mentioned approximations. The fitting procedure provided an estimate for the waiting time $t_{0} \sim 150-400$ as well as for the constant nucleation rate $I$ $\sim 10^{-7}$. The growth velocity $v \sim 0.015$ was measured directly from the various configurations saved during each run.

\section{B. Structure factor}

In his study of first-order phase transitions, Sekimoto [50] derived an expression for the $n$-point correlation function of a system of growing droplets in a nonconserved system. In his work, droplets of the solid phase are assumed to appear 

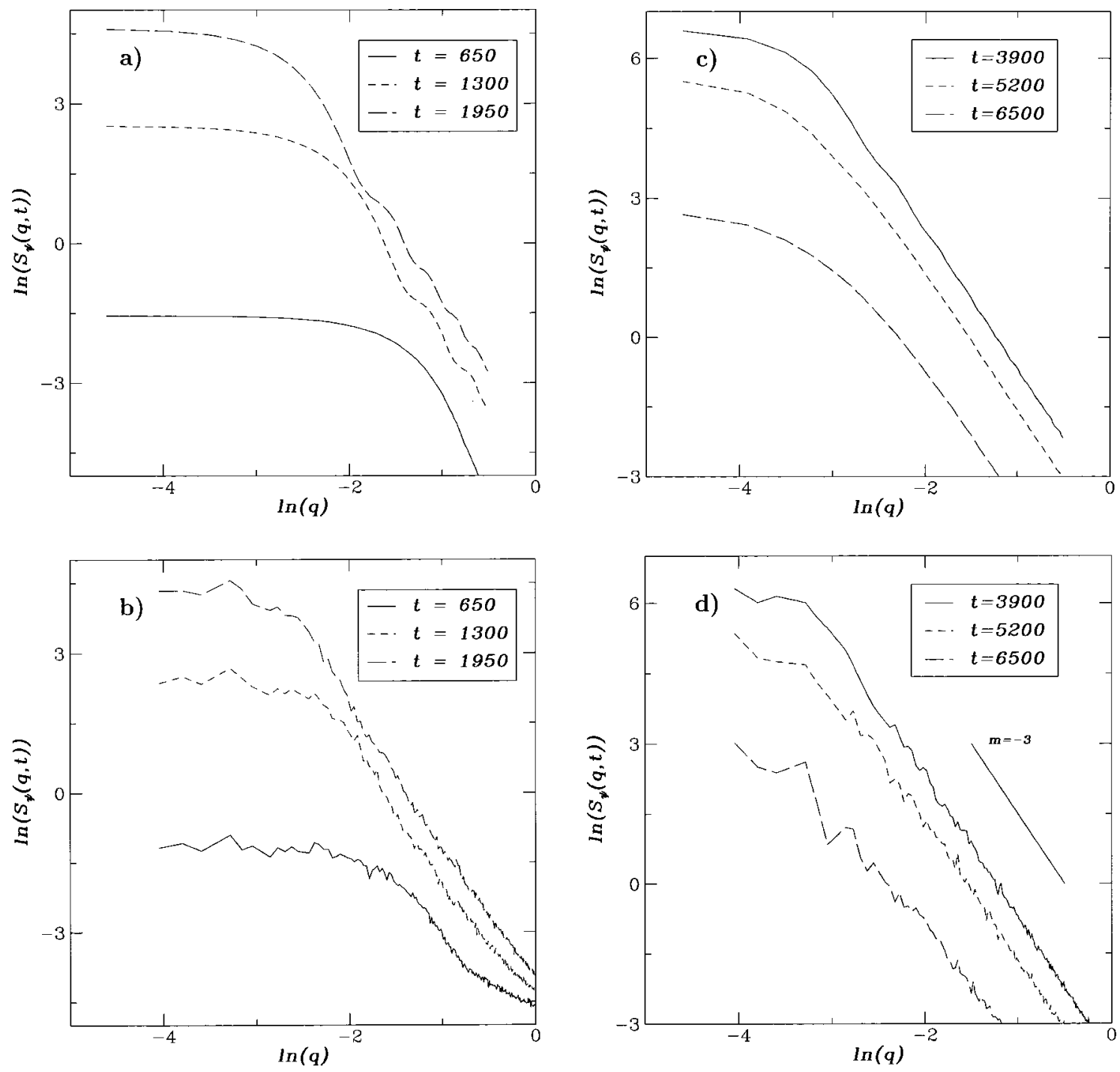

FIG. 11. Wave-number dependence of the $\psi$-field structure factor $S(q, t)$, as predicted by Sekimoto's theory [(a) and (c)], and as measured during simulations [(b) and (d)]. Average composition $c_{0}=0.16$. Theoretical expression with $v=0.016, I=7.2 \times 10^{-8}$, and $t_{0}$ $=143.2$. The power-law behavior at large wave numbers is in agreement with Porod's law, as indicated by the straight line of slope -3 .

randomly at the rate $I$ and are allowed to grow independently despite any overlap between them. The mathematical union of all domains thus formed yields the actual shape of the solid-phase regions. Using this approach, Sekimoto arrived at the following expression for the dynamic structure factor $S(q, t) \equiv\left\langle|\psi(\vec{q}, t)|^{2}\right\rangle$, where $\psi(\vec{q}, t)$ is the Fourier transform of $\psi(\vec{x}, t)$ :

$$
\begin{aligned}
S(q, t)= & \frac{2 \pi\left(2 v t^{\prime}\right)^{2}}{(\Delta \psi)^{2}} e^{-(2 / 3) \pi I v^{2} t^{\prime 3}} \\
& \times \int_{0}^{1} d y\left[e^{I v^{2} t^{\prime 3} \Psi(y)}-1\right] y J_{0}(b y),
\end{aligned}
$$

where $b=2 v t^{\prime} q, t^{\prime}=t-t_{0}, \Delta \psi=\psi_{S}-\psi_{L}, J_{0}$ is the zeroth order Bessel function of the first kind, and $\Psi$ is given by

$$
\Psi(y)=\frac{2}{3}\left[\cos ^{-1}(y)-2 y \sqrt{1-y^{2}}+y^{3} \ln \left(\frac{1+\sqrt{1-y^{2}}}{y}\right)\right]
$$

for $0<y<1$, where $y \equiv r / 2 v t^{\prime}$, and $\Psi=0$ otherwise. Using the trapezoidal rule, the integral in Eq. (16) was evaluated numerically for a variety of times and wave numbers. Both the predicted and measured structure factors are shown on a log-log plot in Fig. 11. The linear regime observed at large $q$-values is in agreement with Porod's law [51,52] which states that, for systems in which the interface thickness is much smaller than the average domain size, $S(q) \sim q^{-(d+1)}$ for large $q$. This law is expected to break down both on scales comparable to the average domain size at small $q$ and also in the very early stages of growth when the interface thickness is comparable to the size of the droplets. The structure factor $S(q, t)$ is also plotted against $t$ in Fig. 12. For all wave numbers, the scenario is identical: the scattering intensity slowly rises from zero as solid droplets start appearing in an otherwise uniform liquid and reaches a maximum around the half-crystallization time $t_{1 / 2}$, defined by $X\left(t_{1 / 2}\right)=1 / 2$, before decreasing again and ultimately vanishing as the system evolves into a uniform solid. The oscillations observed in both the numerical data and the predicted curves correspond to harmonics of the Bessel function $J_{0}(b y)$ in Eq. (16). Since 

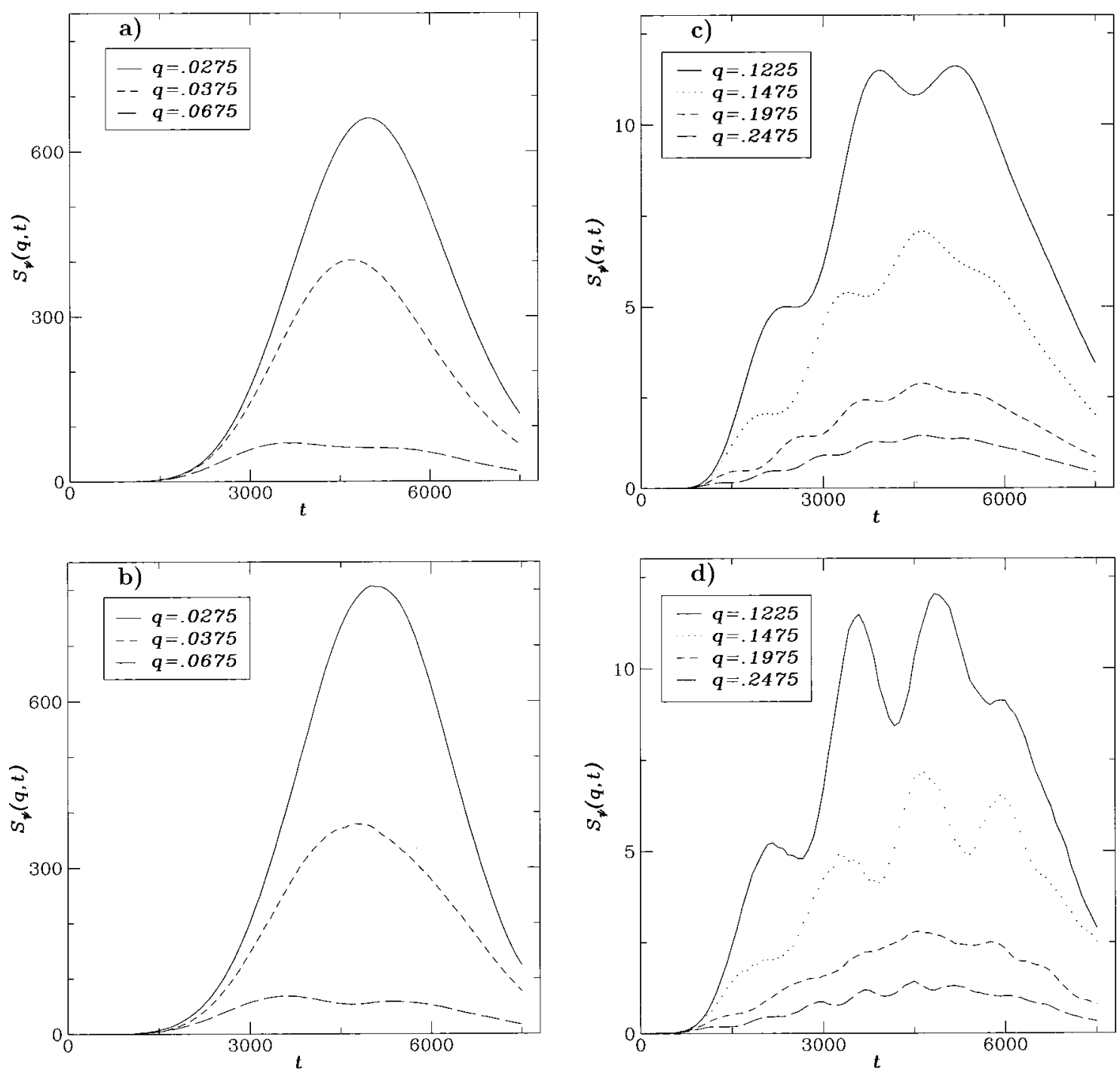

FIG. 12. Time dependence of the $\psi$-field structure factor $S(q, t)$, as predicted by Sekimoto's theory [(a) and (c)], and as measured during simulations $[(\mathrm{b})$ and $(\mathrm{d})]$. Average composition $c_{0}=0.08$. Theoretical expression with $v=0.015, I=3.408 \times 10^{-7}$, and $t_{0}=238.8$.

the spherical droplets eventually coalesce to form domains of arbitrary shape, these oscillations disappear during the late stages of the solidification process.

In summary, the results presented above confirm that Sekimoto's analysis of first-order phase transitions applies to the liquid-solid transformation involved in isothermal eutectic growth. However, a good understanding of the process also requires a description of the changes in composition taking place as the system solidifies. These changes can be attributed to three different growth mechanisms which are now examined.

\section{Growth mechanisms}

The initial droplets formed are usually referred to as primary crystals which typically consist of the solid phase with composition closest to that of the liquid. The initial growth of these droplets is done at the expense of the surrounding liquid, leading to the formation of a boundary layer whose thickness increases with time. An example of such a droplet is shown in Fig. 13(a). The growth of a primary crystal from a $B$-rich liquid, for example, is limited by the transport of $B$ atoms from the melt to the interface. This diffusion-limited process leads to the droplet radius growing as $t^{1 / 2}$. Hence the structure factor associated with the composition field $S_{c}(q, t) \equiv\left\langle|c(\vec{q}, t)|^{2}\right\rangle$ must exhibit a peak at some wave number $q_{m} \propto t^{-1 / 2}$ : the peak and its first harmonic can be seen in Fig. 13(a). Eventually, the interface becomes unstable and domains of the opposite phase simultaneously form along the interface. This event is associated with the appearance of a second peak in the structure factor as in Fig. 13(b). As the wavelength of the lamellar pattern increases with the size of the droplet, this second peak moves to smaller $q$ [Fig. 13(c)]. Its motion eventually stops with new lamellae appearing as the droplet grows.

This is the lamellar growth regime illustrated in Fig. 14. Here the $c$-field configurations shown represent the time evolution of an initial solid seed growing in an environment of eutectic composition $c_{0}=0$. The temperature was set to $\Delta T=-0.14$ and thermal fluctuations suppressed. The structure emerging inside the growing solid consists of alternating lamellae of the $A$ - and $B$-rich phases extending radially from the center of the droplet. As mentioned above, the width of 

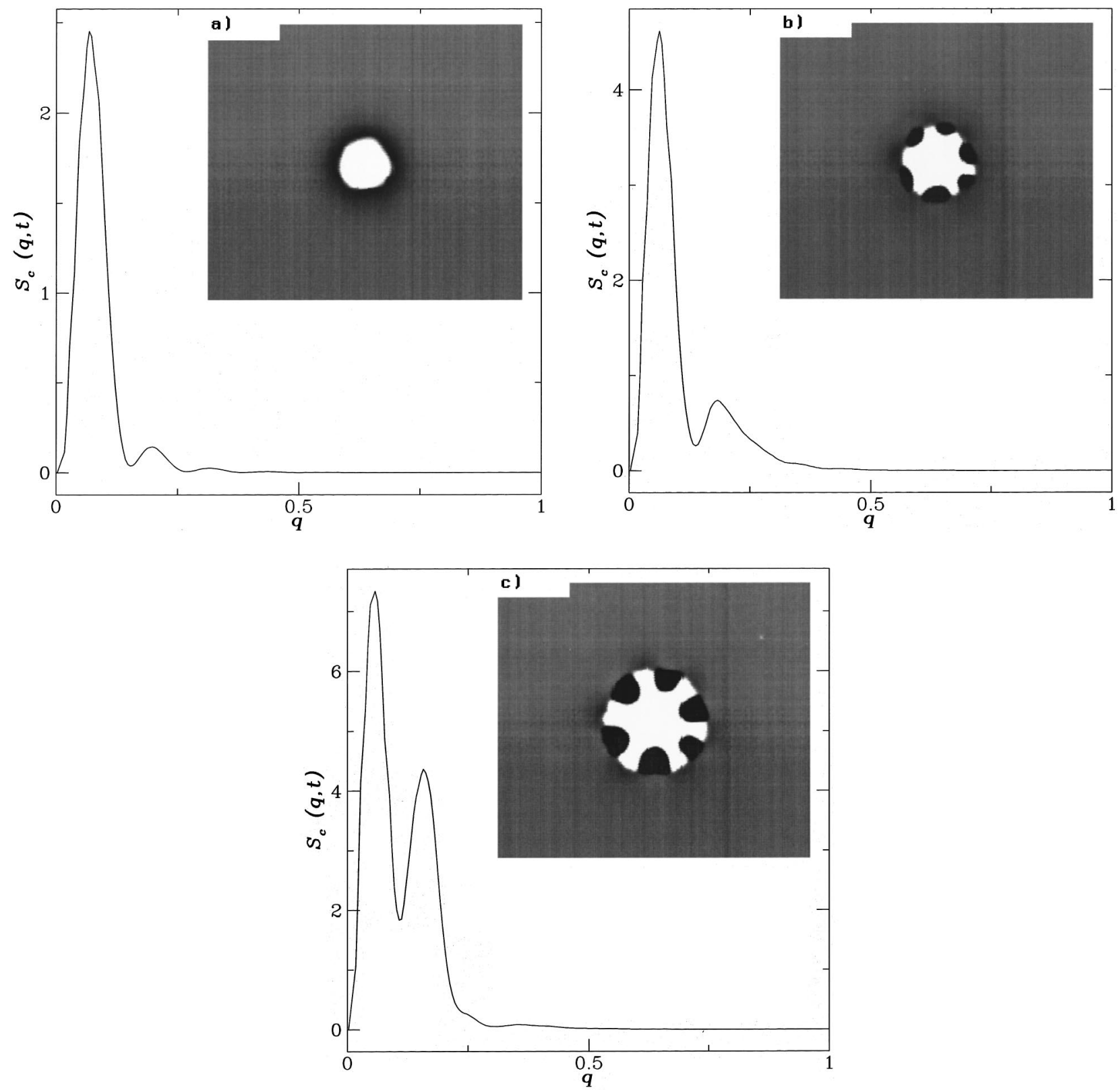

FIG. 13. Transition from diffusion-limited to lamellar growth, as seen from changes in either the system's configuration (insets) or the $c$-field structure factor. The parameter set $\left(K_{\psi}, \alpha, \beta, w\right)=(1 / 8,0.15,0.15,0)$ was used. Thermal fluctuations of magnitude 0.22 were also included. Times are (a) $t=2000$, (b) $t=3000$, and (c) $t=4500$.

the lamellae is expected to be time independent, with new ones appearing as the length of the interface increases. Hence the structure factor should exhibit a peak at some fixed wave number $q_{m}$ with a rescaled height $S_{m} \equiv S_{c}\left(q_{m}, t\right) /\left\langle c^{2}\right\rangle$ proportional to the size of the growing droplet. Since the liquidsolid interface moves at a constant speed $v, S_{m} \propto t$. As in directional lamellar eutectic growth, the system adjusts to a sudden increase in the undercooling and thus in the growth velocity $v$ either by forming tilt-wave-like structures [Fig. 15(a)], or by reducing its lamellar spacing [Fig. 15(b)].

A final growth mechanism sets in as the solid volume fraction $X(t)$ approaches unity. This final regime corresponds experimentally to eutectoids, where the "liquid" phase of our model corresponds to a glass. Typically, this regime is not expected to be observable in binary alloy eutectics, due to the slow diffusion of $c$ in the solid phases. The $\psi$ field becomes uniform and Eq. (4) reduces to the CahnHilliard-Cook model $[32,33]$ of spinodal decomposition. In this limit, growth follows the Ostwald ripening law $[34,35]$. Dimensional analysis requires $q_{m} / w$, where $w$ is the width of the peak, and $S_{m} q_{m}^{d}$ to be constant. When the average composition $c_{0}$ is very far from the eutectic value $c_{0}=0$, the system consists of a number of droplets of the minority solid embedded in a matrix of the majority solid. Again the average radius of the droplets is expected to grow as $t^{1 / 3}$.

In the simulations presented above, only two of the three growth mechanisms were observed as the droplets coalesced before the onset of the lamellar growth regime. The transition from diffusion-limited growth to spinodal decomposition is shown in Fig. 16 for $c_{0}=0.0$, where the time evolution of $1 / q_{m}$ and of $\left[S_{m} w / q_{m}\right]^{1 / 2}$ clearly show a crossover from one regime to the other. The measured late time expo- 

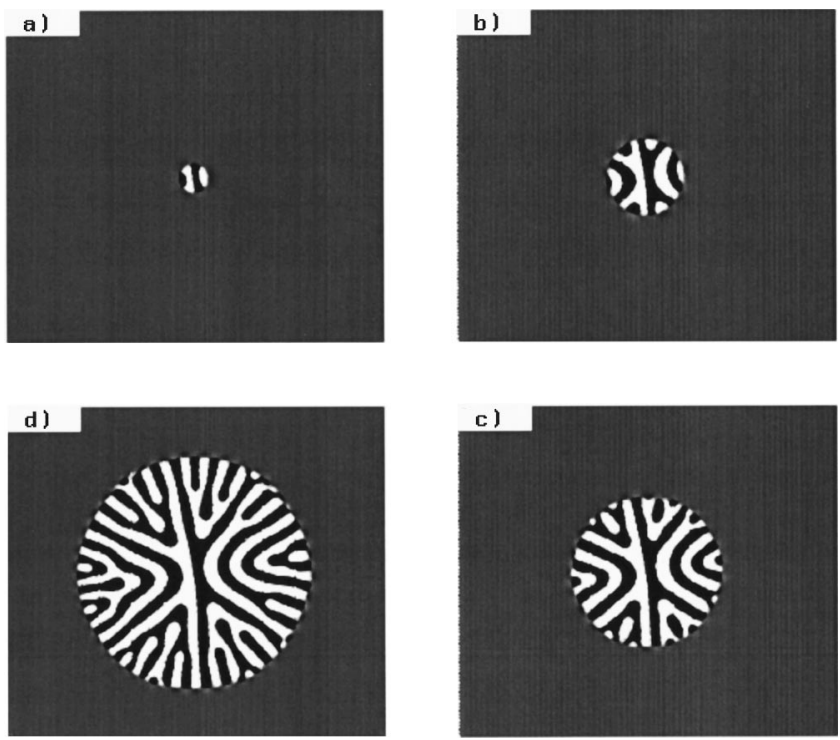

FIG. 14. The lamellar growth regime, as illustrated by the growth of a solid seed in a liquid of eutectic composition. The system size is $512 \times 512$ with $\Delta T=-0.14$. The four configurations displayed are at times $t=1000,3000,5000$, and 7000 [from (a) to (d)].

nent is somewhat smaller than the expected $1 / 3$ and is close to $1 / 4$, with a slight dependence on the fitting procedure used, a result that could be due to the configuration of the system when spinodal decomposition takes over. In any event, it is consistent with other numerical studies of the early stages of spinodal decomposition [36,53]. Furthermore, the late-time plateau seen in the $q_{m} / w$ data indicates that the system is in a scaling regime [54]. The steplike behavior of $q_{m} / w$ for earlier times probably reflects the regular appearance of new phases or "lamellae" inside the solid droplets. As seen in Fig. 13, this can lead to appreciable changes in the structure factor, with $q_{m}$ increasing at the expense of $w$. A recent experiment performed on the eutectoid system $\left(\mathrm{Fe}_{3} \mathrm{~B}\right)+(\mathrm{Fe})$ with $18.5 \% \mathrm{~B}$ [55] confirms the existence of a transition similar to that seen in Fig. 16. The experiment uses in situ methods to obtain time-resolved x-ray scattering patterns at both large and small angles. It is thus sensitive to changes in both electron density from small angle measurements and crystal structure from large angle measurements.

\section{ACKNOWLEDGMENTS}

This work was supported by the Natural Sciences and Engineering Research Council of Canada (M.G.), le Fonds pour la Formation de Chercheurs et l'Aide à la Recherche du Québec (M.G.), NASA Grant No. NAG3-1929 (JMK), and Research Corporation Grant No. CC4181 (K.R.E.). The authors wish to thank Professor Mark Sutton, Dr. Hank Fischer, and Dr. Steve Brauer for numerous helpful discussions.

\section{APPENDIX A: PHASE DIAGRAM CALCULATIONS}

The condition $\delta f(\psi, c) /\left.\delta c\right|_{c_{1}, c_{2}}=\mu_{0}$ determines the bulk composition of any phase present in equilibrium. Close to the eutectic composition, when $c_{L} \approx 0$, the concentration on the liquidus line is
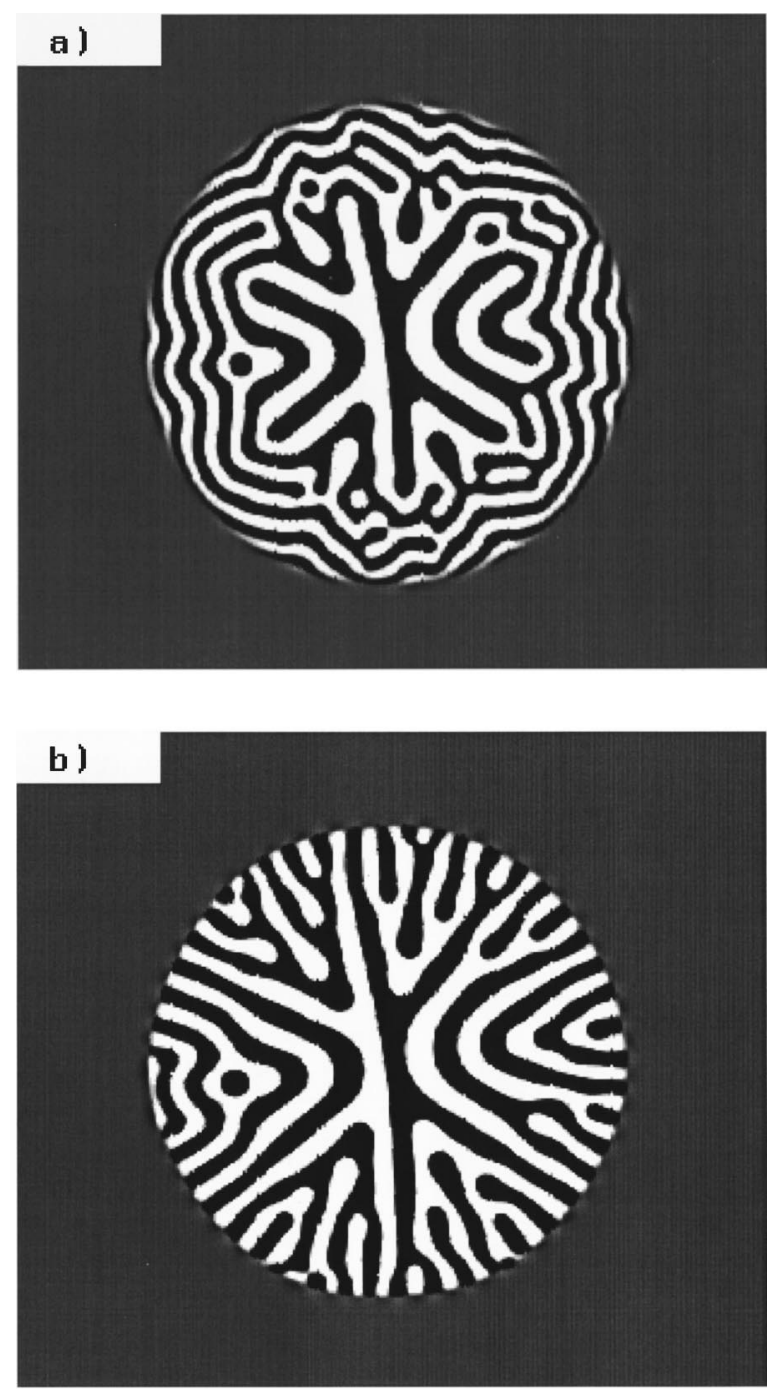

FIG. 15. Changes in the lamellar growth regime following a temperature quench. The configuration displayed in Fig. 14(c) was used as an initial condition. (a) $\Delta T=-0.3$; new lamellae form at an angle with the liquid-solid interface. (b) $\Delta T=-0.17$; new lamellae rapidly form in order to reduce the wavelength of the pattern (as seen by comparing with the bottom-left configuration of Fig. 14).

$$
c_{L} \approx \frac{\mu_{0}}{w+2 \beta(r / a)^{1 / 2}+\alpha \beta \Delta T / r} .
$$

For a solid, a similar procedure yields the roots

$$
\begin{aligned}
c_{S} \approx & \pm\left[\frac{2 \beta(r / a)^{1 / 2}-w-\alpha \beta \Delta T / r}{b-\beta^{2} / r}\right]^{1 / 2} \\
& +\frac{\mu_{0}}{2\left[2 \beta(r / a)^{1 / 2}-w-\alpha \beta \Delta T / r\right]},
\end{aligned}
$$

which is valid provided $w+\alpha \beta \Delta T / r<2 \beta(r / a)^{1 / 2}, b$ $-\beta^{2} / r>0$, and $\mu_{0} \ll 1$. These two expressions, together with Eqs. (6)-(8) are sufficient to calculate the eutectic temperature $\Delta T_{E}$ and the chemical potential $\mu_{0}$. The local slope of both the liquidus and solidus lines can also be evaluated. The eutectic temperature $\Delta T_{E}$ is determined from $f\left(\psi_{L}, c_{L}\right)=f\left(\psi_{S}, c_{E}=0\right)$. The chemical potential $\mu_{0}$ appear- 


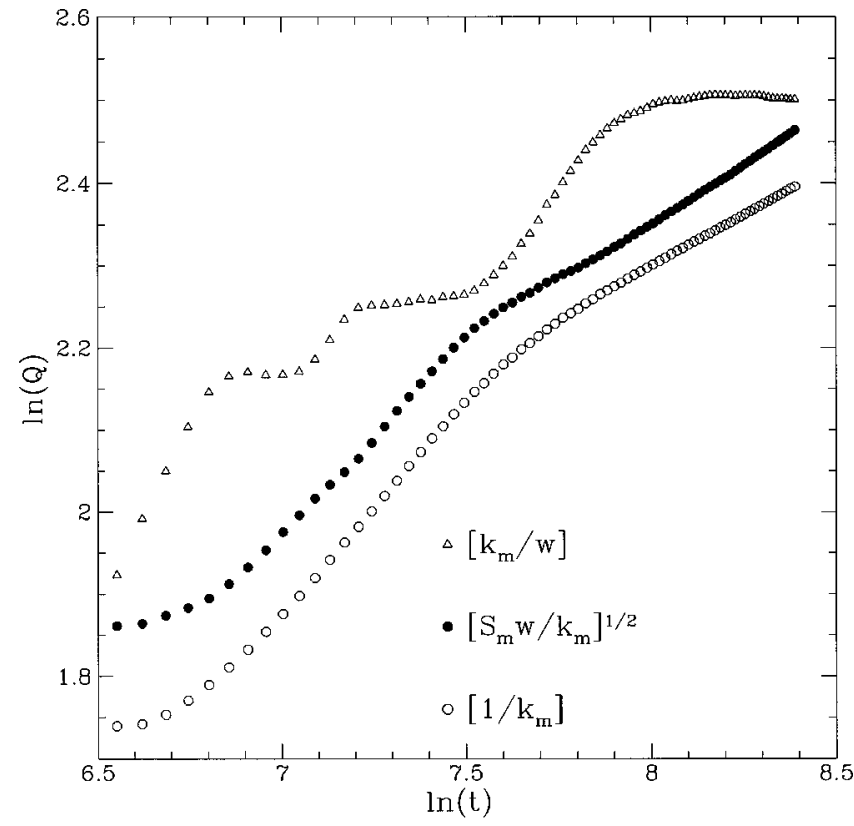

FIG. 16. Transition from diffusion-limited growth to spinodal decomposition.

ing in the expressions for $c_{S}$ and $c_{L}$ can be set to zero as Eq. (8) must be satisfied. Letting $A=w+\alpha \beta \Delta T / r, \quad B$ $=2 \beta(r / a)^{1 / 2}$, and $C=b-\beta^{2} / r$, and keeping terms up to second order in $\Delta T_{E}$ gives $\Delta T_{E}=\left[-f-\left(f^{2}-4 e g\right)^{1 / 2}\right] / 2 a$, where $e=\alpha^{2} \beta^{2} / r^{2}, f=-\left[8 \alpha C(r / a)^{1 / 2}+2 \alpha \beta(B-w) / r\right]$, and, $g=(B-w)^{2}$. The parameters $\alpha, \beta$, and $w$ have been assumed small. A much simpler expression is obtained by going to first order in $\Delta T_{E}$ when

$$
\Delta T_{E} \approx\left[2 \beta(r / a)^{1 / 2}-w\right]^{2} / 8 \alpha b(r / a)^{1 / 2} .
$$

Below the eutectic temperature, $\mu_{0}$ must be identically zero. This follows from the fact that $f(\psi, c)$ is even in $c$ so that two coexisting solids of compositions $\pm c_{S}$ have the same free energy $f\left(\psi_{S}, \pm c_{S}\right)$. Again, from Eq. (8), this implies $\mu_{0}=0$. In the liquid-solid coexistence region, however, the chemical potential is determined by the slope of the common tangent (see Fig. 2). Analytically, again to second order,

$$
\begin{aligned}
\mu_{0}= & \pm\left[\frac{2\left(B^{2}-A^{2}\right)}{B-3 A}\right]\left[\frac{B-A}{C}\right]^{1 / 2} \\
& \times\left[1-\sqrt{1+\frac{B-3 A}{4(B+A)}-\frac{2 \alpha \Delta T(B-3 A) C}{(a / r)^{1 / 2}(B+A)(B-A)^{2}}}\right] .
\end{aligned}
$$

The sign of $\mu_{0}$ depends on the sidearm considered. Restricting the calculation to first order,

$$
\mu_{0} \approx \pm \frac{2 \alpha b^{1 / 2}(r / a)^{1 / 2}}{\left[2 \beta(r / a)^{1 / 2}-w\right]^{1 / 2}}\left(\Delta T-\Delta T_{E}\right)
$$

In the neighborhood of the eutectic temperature, the liquidus and solidus lines are given by Eqs. (A1) and (A2), respectively. To first order in $\Delta T$, their slopes are

$$
\begin{aligned}
m_{L} \equiv \frac{\partial c_{L}}{\partial(\Delta T)} & \approx \frac{1}{\left[2 \beta(r / a)^{1 / 2}+w\right]} \frac{\partial \mu_{0}}{\partial \Delta T} \\
& \approx \pm \frac{2 \alpha b^{1 / 2}(r / a)^{1 / 2}}{\left[2 \beta(r / a)^{1 / 2}-w\right]^{1 / 2}\left[2 \beta(r / a)^{1 / 2}+w\right]}
\end{aligned}
$$

and

$$
\begin{aligned}
m_{S} & \equiv \frac{\partial c_{S}}{\partial(\Delta T)} \approx \frac{1}{2\left[2 \beta(r / a)^{1 / 2}-w\right]} \frac{\partial \mu_{0}}{\partial(\Delta T)} \\
& \approx \pm \frac{\alpha b^{1 / 2}(r / a)^{1 / 2}}{\left[2 \beta(r / a)^{1 / 2}-w\right]^{3 / 2}} .
\end{aligned}
$$

\section{APPENDIX B: SHARP-INTERFACE EQUATIONS}

The sharp-interface equations can be obtained formally by expanding around a planar interface in equilibrium, as described by the equations $\delta \mathcal{F} / \delta \psi=0$ and $\delta \mathcal{F} / \delta c-\mu_{e q}=0$. For concreteness, consider expanding around a liquid-solid interface at the eutectic temperature, with the liquid at the eutectic concentration and the solid at the coexistence concentration. To address phenomena occuring when the interface is gently curved, and when it moves due to a small degree of metastability in one of the phases, we need to make a perturbation expansion in curvature $\kappa$ and velocity $v$, respectively. Of course, these expansions must be in dimensionless quantities, namely, $\kappa \xi$, and $\xi v / D$, where $\xi$ is the interface width, and $D$ is a diffusion constant. As mentioned previously, one must not only require, for example, $\kappa \xi \ll 1$. On general grounds, one must also have $\xi / l_{0} \rightarrow \infty$, where the ultraviolet cutoff $l_{0} \rightarrow 0^{+}$, to obtain the sharp-interface equations.

The analysis follows the standard method of Kawasaki and Ohta [56], which makes use of the projection operator, defined in terms of the one-dimensional planar solution $\psi^{0}(u)$,

$$
\mathcal{P}_{\psi}(\cdots) \equiv \frac{1}{\Delta \psi} \int_{-\infty}^{+\infty} d u \frac{d \psi^{0}}{d u}(\cdots),
$$

where $u$ is the direction normal to the surface. This operator projects the dynamics of the full phase-field model onto that of the surface, in a way which is controlled order by order in the two small parameters $\kappa \xi$ and $\xi v / D$. It is convenient to introduce an orthogonal curvilinear coordinate system $(\vec{x})$ $=(u, \vec{s})$ such that $|\vec{s}|$ is the arc length along the surface. It is also convenient to eliminate the Laplacian from the conservation law for $c$, through use of the Green function defined by $\nabla^{2} G(\vec{x}, 0)=\delta(\vec{x})$.

After formally solving the conserved equation for $c$ through use of the Green function, and expanding all quantities in terms of the small parameters, where lowest order terms corresponding to the one-dimensional planar solution have a superscript " 0 ,', the equations are acted on by $\mathcal{P}_{\psi}$ and $\mathcal{P}_{c}$. To first order these calculations give what are often called the "inner solutions" 


$$
\frac{\sigma_{\psi} v_{n}}{\Gamma_{\psi} K_{\psi}}=\sigma_{\psi} \kappa+\left.\int d u\left(\delta \psi \frac{d c^{0}}{d u}-\delta c \frac{d \psi^{0}}{d u}\right) \frac{\partial^{2} f}{\partial \psi \partial c}\right|^{0}
$$

and

$$
\begin{aligned}
\mu(u & =0) \Delta c+v_{n} \frac{g}{\Gamma_{c}} \\
& =-\sigma_{c} \kappa+\left.\int d u\left(\delta \psi \frac{d c^{0}}{d u}-\delta c \frac{d \psi^{0}}{d u}\right) \frac{\partial^{2} f}{\partial \psi \partial c}\right|^{0},
\end{aligned}
$$

where $\mu(0)$ is the chemical potential at the surface, $\Delta c=c_{L}-c_{S}, \quad \sigma_{\psi} \equiv K_{\psi} \int d u\left(d \psi^{0} / d u\right)^{2}, \quad \sigma_{c}$ $\equiv K_{c} \int d u\left(d c^{0} /\right.$

$d u)^{2}$, and $g=\left[\int_{0}^{\infty} d u\left(c^{0}(u)-c^{0}(\infty)\right)^{2}+\int_{-\infty}^{0} d u\left(c^{0}(u)-c^{0}\right.\right.$ $\left.(-\infty))^{2}\right]+\Delta c \int_{0}^{\infty} d u\left(c^{0}(\infty)-c^{0}(u)\right)$. In this calculation the interface was chosen to be the Gibb's surface such that the excess surface concentration is equal on both sides of the interface, i.e., $\quad \int_{0}^{\infty} d u\left(c^{0}(\infty)-c^{0}(u)\right)=\int_{-\infty}^{0} d u\left(c^{0}(u)-c^{0}\right.$ $(-\infty))=0$.

In obtaining Eq. (B3), the result

$$
v_{n} \Delta c=\left.D_{L} \frac{\partial c}{\partial u}\right|_{L}-\left.D_{S} \frac{\partial c}{\partial u}\right|_{S}
$$

was used, where the diffusion constant is $D$ $=\left.\Gamma_{c}\left(\partial^{2} f / \partial c^{2}\right)\right|_{c_{e q}}$ which gives $D_{L}=\Gamma_{c}\left[w-2 \beta \psi_{L}+3 b c_{L}^{2}\right]$ and $D_{S}=\Gamma_{c}\left[w-2 \beta \psi_{S}+3 b c_{S}^{2}\right]$ for the free energy discussed in this paper. This result can be easily obtained by integrating over the equation of motion for $c$ to lowest order in the small parameters, i.e.,

$$
v_{n} \frac{\partial c}{\partial u}=\Gamma_{c} \frac{\partial^{2} \mu}{\partial u^{2}}
$$

from the solid to liquid phase.

Subtracting Eq. (B3) from Eq. (B2), and expanding the chemical potential to lowest order in concentration and temperature, gives [57]

$$
\frac{\delta c(0)}{\Delta c_{m i s}}=d_{0} \kappa+\frac{\zeta}{l_{T}}+\frac{d_{0} v_{n}}{\sigma}\left(\frac{g}{\Gamma_{c}}-\frac{\sigma_{\psi}}{\Gamma_{\psi} K_{\psi}}\right),
$$

where $\xi$ is the distance between the interface and the eutectic temperature, $\quad d_{0}=2 \sigma /\left[(\partial \mu / \partial c)\left(\Delta c_{m i s}\right)^{2}\right], l_{T}$ $=m \Delta c_{m i s} / G, \quad m=\partial T / \partial c$, and $\sigma=\sigma_{c}+\sigma_{\psi}$.

The last element of the sharp-interface formulation is a condition of mechanical equilibrium imposed at the junction of the three phases. Specifically, the net force acting on the point of contact between the liquid and the two solids $A$ and $B$ must be zero in equilibrium. Hence $\sigma_{L A} \cos \theta=\sigma_{L B} \cos \chi$ and $\sigma_{L A} \sin \theta+\sigma_{L B} \sin \chi=\sigma_{A B}$, where $\theta$ and $\chi$ are defined defined in Fig. 17, $\sigma_{L A}$ is the surface tension associated with the interface between the liquid phase, and the solid $A$ phase, and so on. These conditions take a simpler form in the present case as the invariance of $\mathcal{F}$ under $c \rightarrow-c$ implies $\sigma_{L A}=\sigma_{L B} \equiv \sigma_{L S}$. Thus $\theta=\chi$ and

$$
2 \sigma_{L S} \sin \theta=\sigma_{A B} .
$$

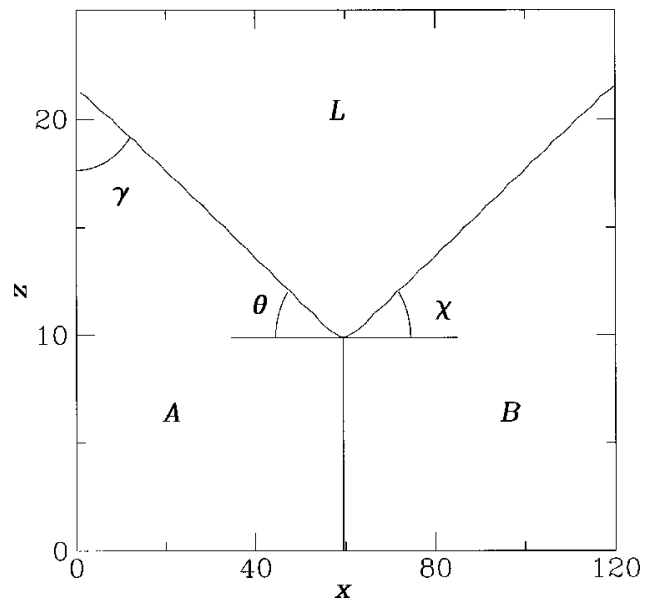

FIG. 17. Condition of mechanical equilibrium where the three phases meet. The equilibrium angle $\theta=11.4^{\circ}$ was obtained using the procedure described at the end of Appendix B. The $L-A$ and $L-B$ interfaces correspond to points where $\psi=0$, while the $A-B$ interface is associated with points where $c=0$.

A general expression for the surface tensions can be obtained from the mean-field equilibrium conditions $\delta \mathcal{F} / \delta \psi$ $=0$ and $\delta \mathcal{F} / \delta c=\mu_{0}$ where $\mu_{0}=0$ at $T=T_{E}$. Assuming that both $\psi$ and $c$ depend only on the coordinate $u$ locally normal to the interface, the first condition gives $\partial f(\psi, c) / \partial \psi$ $=K_{\psi} d^{2} \psi / d u^{2}$, or, on integrating,

$$
f(\psi, c)=\frac{K_{\psi}}{2}\left(\frac{d \psi}{d u}\right)^{2}+h(c) .
$$

The integration constant $h(c)$ can be determined from minimizing with respect to $c$, giving $h(c)=\left(K_{c} / 2\right)(d c / d u)^{2}$. Using this result for $f$ gives $\mathcal{F}=\left(2 \int d u f\right) \int d s$, from which the surface tension can be identified as

$$
\sigma=2 \int d u f=\sigma_{\psi}+\sigma_{c}
$$

Evaluation of $\sigma_{c}$ is possible for the $A-B$ interface. As both phases are solid, $\psi$ is first replaced with its mean-field expression $\psi_{S}$ given in Eq. (7). With this form, one obtains

$$
K_{c} \frac{d^{2} c}{d u^{2}}+\left[2 \beta\left(\frac{r}{a}\right)^{1 / 2}-\frac{\alpha \beta \Delta T}{r}-w\right] c-\left(b-\frac{\beta^{2}}{r}\right) c^{3}=0
$$

the solution to which is $c(u)=\left[\left(2 \beta(r / a)^{1 / 2}-\alpha \beta \Delta T / r\right.\right.$ $\left.-w) /\left(b-\beta^{2} / r\right)\right]^{1 / 2} \tanh (u / \xi)$, where the interface thickness $\xi \equiv\left[2 K_{c} /\left(2 \beta(r / a)^{1 / 2}-\alpha \beta \Delta T / r-w\right)\right]^{1 / 2}$. Then one obtains $\sigma_{c}=4 \sqrt{K_{c}}\left[2 \beta(r / a)^{1 / 2}-\alpha \beta \Delta T / r-w\right]^{3 / 2} / 3 \sqrt{2}\left(b-\beta^{2} / r\right)$.

These results were compared to numerical estimates from simulations. For example, for the parameter set $\left(K_{c}, r, a, b, w, \alpha, \beta\right)=(1,1,1,1,0,0.30,0.30)$ at the eutectic temperature $\Delta T_{E}, \sigma_{c}=0.465$. As variations in the $\psi$ field are very small, their contribution to the surface tension can be neglected. Thus $\sigma_{A B} \approx \sigma_{c}=0.465$. The remaining surface tension $\sigma_{L S}$ is obtained by simultaneously solving the equations for $\partial f / \partial \psi$ and $\partial f / \partial c$. This was done numerically in one dimension, and, for a liquid of eutectic composition, yielded the values $\sigma_{c}=0.104$ and $\sigma_{\psi}=1.018$ or $\sigma_{L S}$ 
$=1.122$. Finally, from Eq. (B7), the equilibrium angle was found to be $\theta=11.9^{\circ}$. This value was compared to a simulation estimate obtained by integrating Eqs. (3) on a 120 $\times 30$ square lattice. The derivative of both fields in the $z$ direction was set to zero at the top and bottom of the simulation box (Fig. 17). A constant angle $\gamma=90^{\circ}-\theta$ betweenthe interfaces $L-A$ and $L-B$ and the walls of the system was imposed. Using the grid parameters $\Delta x=1.0$ and $\Delta t$ $=0.025$, the procedure yielded the value $\theta=11.4^{\circ}$, in good agreement with the estimate above of $\theta=11.9^{\circ}$. Note that this good agreement involves a nonzero thickness of the interface.
[1] G. Ahlers, D. S. Cannell, and V. Steinberg, Phys. Rev. Lett. 54, 1373 (1985).

[2] M. S. Heutmaker, P. N. Fraenkel, and J. P. Gollub, Phys. Rev. Lett. 54, 1369 (1985).

[3] S. W. Morris, E. Bodenshatz, D. S. Cannell, and G. Ahlers, Phys. Rev. Lett. 71, 2026 (1993).

[4] H. W. Xi, J. D. Gunton, and J. Viñals, Phys. Rev. Lett. 71, 2030 (1993).

[5] M. E. Glicksman, R. J. Schaefer, and J. D. Ayers, Metall. Trans. A 7, 1747 (1976).

[6] J. S. Langer, Rev. Mod. Phys. 52, 1 (1980).

[7] S. C. Huang and M. E. Glicksman, Acta Metall. 29, 701 (1981); 29, 717 (1981).

[8] M. E. Glicksman, Mater. Sci. Eng. 65, 45 (1984).

[9] J. C. LaCombe, M. B. Koss, V. E. Fradkov, and M. E. Glicksman, Phys. Rev. E 52, 2778 (1995)

[10] B. Caroli, C. Caroli and B. Roulet, in Solids Far From Equilibrium, edited by G. Godrèche (Cambridge University Press, Cambridge, 1992).

[11] J. B. Smith, J. Comput. Phys. 39, 112 (1981).

[12] R. Almgren, J. Comput. Phys. 106, 337 (1993).

[13] G. J. Fix, in Free Boundary Problems: Theory and Applications, edited by A. Fasano and M. Primicerio (Pitman, Boston, 1983), Vol. II.

[14] J. B. Collins and H. Levine, Phys. Rev. B 31, 6119 (1985).

[15] K. R. Elder, F. Drolet, J. M. Kosterlitz, and M. Grant, Phys. Rev. Lett. 72, 677 (1994).

[16] See also related work by A. A. Wheeler, W. J. Boettinger, and G. B. McFadden, Phys. Rev. A 45, 7424 (1992); A. Karma, Phys. Rev. E 39, 2245 (1994); A. A. Wheeler, G. B. McFadden, and W. J. Boettinger, Proc. R. Soc. London, Ser. A 452, 495 (1996); I. Steinbach, F. Pezzola, B. Nestler, M. Seesselberg, R. Prieler, G. J. Schmitz, and J. L. L. Rezende, Physica D 94, 135 (1996).

[17] K. R. Elder, J. D. Gunton, and M. Grant, Phys. Rev. E 54, 6476 (1996).

[18] J. P. Chilton and W. C. Winegard, J. Inst. Met. 89, 162 (1961).

[19] A. S. Yue, Trans. Metall. Soc. AIME 224, 1010 (1962).

[20] K. A. Jackson and J. D. Hunt, Trans. Metall. Soc. AIME 236, 843 (1966).

[21] K. A. Jackson and J. D. Hunt, Trans. Metall. Soc. AIME 236, 1129 (1966).

[22] J. S. Langer, Phys. Rev. Lett. 44, 1023 (1980).

[23] V. Datye and J. S. Langer, Phys. Rev. B 24, 4155 (1981).

[24] A. Karma, Phys. Rev. Lett. 59, 71 (1987).

[25] K. Kassner and C. Misbah, Phys. Rev. Lett. 66, 445 (1991); Phys. Rev. A 44, 6513 (1991).

[26] K. Kassner and C. Misbah, Phys. Rev. Lett. 65, 1458 (1990); Phys. Rev. A 44, 6533 (1991).

[27] See, for example, the discussion of free energy functionals given by N. Goldenfeld, Lectures on Phase Transitions and the Renormalization Group (Addison-Wesley, Reading, MA, 1992).

[28] Note that the mobilities for $\psi$ and $c$ are $\Gamma \neq \Gamma(c, \psi)$. The diffusivities in different phases can be different due to different susceptibilities in the phases, as shown in Appendix B. Hence the degree to which transport is different in different phases arises naturally from the mesoscopic Ginzburg-Landau free energy, the identification of the dynamical variables ( $c$ and $\psi$ herein), and the minimal dynamical Langevin equations consistent with conservation laws and fluctuation-dissipation relations. This is in contrast to imposing different transport in different phases: In particular, the conditions under which one may take, for example, $\Gamma_{c} \nabla^{2} \rightarrow \vec{\nabla} \cdot\left(\Gamma_{c}(c, \psi) \vec{\nabla}\right)$, consistent with fluctuation-dissipation relations, and the chosen thermodynamic free energy $-k_{B} T \ln \Sigma_{c, \psi} \exp -\mathcal{F} / k_{B} T$, are not obvious.

[29] R. Kobayashi, Physica D 63, 410 (1993).

[30] K. R. Elder, M. Grant, N. Provatas, and J. M. Kosterlitz (unpublished).

[31] This assumes both phases have a low entropy of melting. Eutectics for which this assumption does not hold form either irregular structures or crystalline facets [20].

[32] J. W. Cahn and J. E. Hilliard, J. Chem. Phys. 28, 258 (1958).

[33] H. E. Cook, Acta Metall. 18, 297 (1970).

[34] I. M. Lifshitz and V. V. Slyozov, J. Phys. Chem. Solids 19, 35 (1961).

[35] J. H. Yao, K. R. Elder, H. Guo, and M. Grant, Phys. Rev. B 47, 14110 (1993); Physica A 204, 770 (1994).

[36] T. R. Rogers, K. R. Elder, and R. C. Desai, Phys. Rev. B 37, 9638 (1988).

[37] A. J. Bray, Adv. Phys. 43, 357 (1994).

[38] G. Faivre, S. De Cheveigne, C. Guthmann, and P. Kurowski, Europhys. Lett. 9, 779 (1989).

[39] G. Faivre and J. Mergy, Phys. Rev. A 46, 963 (1992).

[40] J. W. Rutter and B. Chalmers, Can. J. Phys. 31, 15 (1953).

[41] W. A. Tiller, K. A. Jackson, J. W. Rutter, and B. Chalmers, Acta Metall. 1, 428 (1953).

[42] K. A. Jackson and J. D. Hunt, Acta Metall. 13, 1212 (1965).

[43] D. Walton, W. A. Tiller, J. W. Rutter, and W. C. Winegard, Trans. AIME 203, 1023 (1955).

[44] W. W. Mullins and R. F. Sekerka, J. Appl. Phys. 34, 323 (1963); 35, 44 (1964).

[45] B. Grossmann, K. R. Elder, M. Grant, and J. M. Kosterlitz, Phys. Rev. Lett. 71, 3323 (1993).

[46] Of course, an $A$-rich liquid nucleates the $A$-rich solid first.

[47] A. N. Kolmogorov, Bull. Acad. Sci. USSR 3, 335 (1938).

[48] W. A. Johnson and R. F. Mehl, Trans. Am. Inst. Min., Metall. Pet. Eng. 135, 416 (1939).

[49] M. Avrami, J. Chem. Phys. 7, 1103 (1939); 8, 212 (1940): 9, 177 (1941).

[50] K. Sekimoto, Physica A 135, 328 (1986). 
[51] G. Porod, Kolloid-Z. 124, 83 (1952); 125, 125 (1952).

[52] G. Porod, in Small Angle X-ray Scattering, edited by O. Glatter and O. Kratky (Academic Press, New York, 1982).

[53] Y. Oono and S. Puri, Phys. Rev. Lett. 58, 836 (1987).

[54] The presence of a single relevant length scale $L$ implies that both $k_{m}$ and $w$, which scale as $L^{-1}$, have the same time dependence and that their ratio is constant.

[55] H. Fischer, S. Brauer, M. Sutton, J. Ström-Olsen, B. Stephen- son, and U. Koster (private communication).

[56] K. Kawasaki and T. Ohta, Prog. Theor. Phys. 67, 147 (1982); K. Kawasaki and T. Ohta, ibid. 68, 129 (1982); K. Kawasaki and T. Ohta, Physica A 118, 175 (1983).

[57] See also, for example, G. Caginalp and W. Xie, Phys. Rev. E 48, 1897 (1993); A. Karma and W.-J. Rappel, ibid. 57, 4323 (1998). 